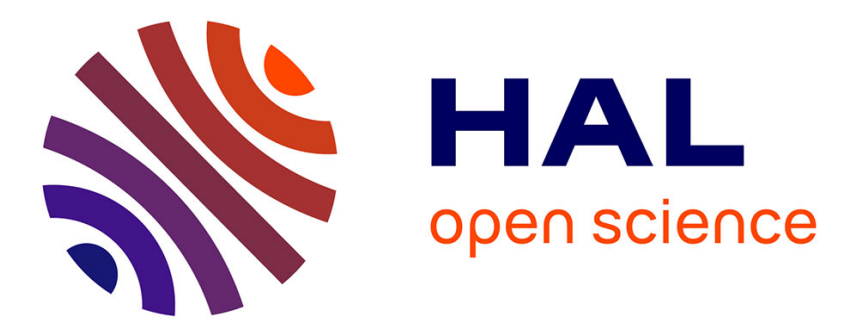

\title{
Human to humanoid motion conversion for dual-arm manipulation tasks
}

Marija Tomić, Christine Chevallereau, Kosta Jovanović, Veljko Potkonjak, Aleksandar Rodić

\section{- To cite this version:}

Marija Tomić, Christine Chevallereau, Kosta Jovanović, Veljko Potkonjak, Aleksandar Rodić. Human to humanoid motion conversion for dual-arm manipulation tasks. Robotica, 2018, 36 (8), pp.11671187. 10.1017/S0263574718000309 . hal-01819258

\section{HAL Id: hal-01819258 \\ https://hal.science/hal-01819258}

Submitted on 20 Jun 2018

HAL is a multi-disciplinary open access archive for the deposit and dissemination of scientific research documents, whether they are published or not. The documents may come from teaching and research institutions in France or abroad, or from public or private research centers.
L'archive ouverte pluridisciplinaire HAL, est destinée au dépôt et à la diffusion de documents scientifiques de niveau recherche, publiés ou non, émanant des établissements d'enseignement et de recherche français ou étrangers, des laboratoires publics ou privés. 


\section{Human to humanoid motion conversion for dual-arm manipulation tasks}

Marija Tomić $\dagger^{*}$, Christine Chevallereau $\ddagger$, Kosta Jovanović $\uparrow$, Veljko Potkonjak $\dagger$, and Aleksandar Rodić*

$\uparrow$ School of Electrical Engineering, University of Belgrade, Bulevar kralja Aleksandra 73, 11000 Belgrade, Serbia

†CNRS, LS2N, Ecole Centrale de Nantes, 1, rue de la No, 44321 Nantes, France

*Robotics Laboratory, IMP, Volgina 15, 11000 Belgrade, Serbia

\section{SUMMARY}

A conversion process for the imitation of human dual-arm motion by a humanoid robot is presented. The conversion process consists of an imitation algorithm and an algorithm for generating human-like motion of the humanoid. The desired motions in Cartesian and joint spaces, obtained from the imitation algorithm, are used to generate the human-like motion of the humanoid. The proposed conversion process improves existing techniques and is developed with the aim to enable imitating of human motion with a humanoid robot, to perform a task with and/or without contact between hands and equipment. A comparative analysis shows that our algorithm, which takes into account the situation of marker frames and the position of joint frames, ensures more precise imitation than previously proposed methods. The results of our conversion algorithm are tested on the robot ROMEO through a complex “open/close drawer" task.

KEYWORDS: Motion capture system; dual arm manipulation; imitation algorithms; humanoid robot, motion planning.

\section{Introduction}

The imitation process is an interesting and fast method for teaching robots new motion skills. The motion 
learning process is based on the repetition of observed motions. Once a human observes a task being carried out for the first time, they are usually capable of immediately performing the same action. In robotics, this process is more complex and can be interpreted in different ways, such as: the imitation learning process and the motion imitation process [1]. The imitation learning process is aimed at understanding the characteristics of human motions, based on data collected from multiple observations of the demonstrated action. On the other hand, the motion imitation process is based on the imitation of human movements without any analysis of the characteristics of the human motion. In this paper we consider the latter approach and propose an algorithm based on how the robot imitates human motion, by utilizing recorded data obtained from a marker-based motion capture system.

Today, there are numerous techniques for recording and analyzing human motions. Human motions can be tracked by a number of different motion capture systems, such as inertial, optical, magnetic, acoustic or mechanical. The type of motion capture system used depends on the volume of measurements taken, the required resolution, the characteristics of the motion recorded and the environment in which the motion is performed. Acoustic [2], inertial [3] and magnetic [4] motion capture systems are able to record outdoor and indoor human motions. The most commonly used techniques for recording human movement are marker-based and marker-less motion capture systems. Comparative analyses of different motion capture systems are given in references [5-7]. In order to record a 3D model of human motion, including the position and orientation of the required segments and joints, in this research we used an ART marker-based motion capture system.

The imitation process can be defined in Cartesian space and/or joint space [8]. In Cartesian space, the motions of the hands, head, or feet are recorded and a geometric inverse model of the humanoid can be used to achieve the task. In joint space, the objective is to enable the robot to replicate joint motions of the human, following the human configuration. This last objective is conventionally used for the imitation 
process and allows for human-like behavior, especially in the presence of a redundant robot. If a human (or a robot) has to achieve the same task in Cartesian space as another human of different body size, they have to modify the motion of the joints while keeping the end-effector (hands, feet or head) in the same Cartesian position. Since the human (or robot) is a kinematic redundant structure, the same end-effector trajectories are feasible for different joint trajectories. The main idea of the research was to achieve these joint trajectories so they fit the motion of human joints as much as possible while keeping the required end-effector motion in Cartesian space. Therefore, joint trajectory tracking was the object of optimization, while end-effector trajectory tracking was an imposed constraint in the optimization process. It should be noted that depending on the task being analyzed, various components in Cartesian space can be taken into account (position, or position and orientation of the hands). In the case of a task that involves contact between hands and equipment, the position and orientation of the hand should be included as a constraint in the optimization process, whereas in a task without contact between hands and equipment, following of the position of the hands is required. In short, to imitate a dual-arm human motion, where the robot interacts with the environment using its hands and exhibits human-like motion behavior, the imitation process needs to include mimicking of human motion in the joint and Cartesian spaces.

The human motion imitation problem can be solved at the kinematic or the dynamic level. In Ott et al. [9], the robot imitated human motions by using dynamic equations. Based on measurements of marker positions, they define a Cartesian control approach to real-time imitation of a human's upper body. Virtual springs connected the measured marker positions and corresponding points on the humanoid robot. Since additional difficulties arise in the process of motion imitation by a humanoid robot, such as joint velocity and torque limits, Suleiman et al. [10] also use dynamic equations to formulate a recursive optimization algorithm for imitating human motion. It permits the imitation of upper-body human motion by using the physical capabilities (joint, velocities, torques) of a humanoid robot. Huang et al. [11] present an algorithm that provides humanoid motion which is very similar to human motion and satisfies kinematic 
constraints and dynamic stability. Jamisola et al. [12] show modular task-space dynamic formulations for a dual arm controlled as a single manipulator. They used existing kinematic and dynamics models of each of the stand-alone manipulators to arrive at the overall dynamics of the single end-effector dual-arm. The treatment of a dual-arm as a single end-effector manipulator through the use of the relative Jacobian affords a drastic increase in the null-space dimension and lesser constraints in the task space. This approach was tested for dual-arm manipulation tasks which did and did not require coordination between hands.

The human motion imitation problem is addressed at the kinematic level by Ude et al. [13, 14]. They propose a method for transforming the recorded 3D position of the markers into high dimensional trajectories of the humanoid robot joints based on twist representation. The human body was modeled as a scaled model of the humanoid robot. They established relationships between the motion of the robot's joints and the motion of the markers by using B-spline wavelets and large-scale optimization techniques. The method was applied offline to a humanoid robot called DB. Ayusawa et al. [15, 16] propose a gradient-based method for simultaneous identification of the geometric parameters of a human skeletal model and calculation of inverse kinematics by using information about the recorded positions of markers. In order to define the geometric parameters, as segment lengths, and the positions of the markers relative to the robot segments, they introduced virtual translation of joints. In the present research, the problem of human motion imitation was studied at the kinematic level.

According to the state of the art of the imitation process, we additionally explored:

- Imitation of the upper-body human motion by the humanoid, where the motion consists of phases without and/or with hand contact with the environment and a transition strategy between these two types of motion. 
- An analytical imitation algorithm based on the Jacobian matrix (instead of the standard optimization algorithm presented in our previous research [17]), which is capable of real time extraction of Cartesian motions and joint motions that can be used by the humanoid.

In the present research, human-to-humanoid motion conversion is divided into two parts. In the first part, we used the information provided by the motion capture system and analytically defined the imitation algorithm to acquire the desired motion of the humanoid in the task and joint spaces. The algorithm is based on the markers positioned on a scaled model of the humanoid (virtual markers), which follow the motion of markers (real markers) placed on the human. The intermediate use of a scaled model of the humanoid presented by Ude et al. [14] permits the size of the robot to be adapted to the size of the human that has accomplished the task and thus to record the coherent joint and Cartesian motions. Since the task of the imitation algorithm proposed in the present paper is to generate a motion where the hands and the environment are in contact, precise imitation of the hand motions is important. This imitation algorithm incorporated not only marker motion but also joint motion measurement, in order to increase the robot's imitation accuracy. Instead of twists in our kinematic model, the modified Denavit and Hartenberg (DH) convention was used to simplify modeling [18]. The imitation algorithm is based on the kinematic structure of the humanoid and can be used in real time.

In the second part of the conversion, the motion imitation of the scaled robot model was used to generate a human-like motion on the robot ROMEO in its real size $(1.40 \mathrm{~m}$ tall) and in the same environments. The strategy differed depending on whether a contact with the environment did or did not exist during the studied phase of motion. When there was contact with the environment, the hand motions were defined to achieve the contact and the robot hand motions had to be the same as those of the human. During the phase without contact, priority was given to motion that appeared visually close to human motion and was based on similar joint motions of the humanoid robot with respect to the human. Hand motions can be modified 
since they are not constrained by equipment. As such, if contact motion is observed, hand motion in Cartesian space is perceived as a constraint while joint positions in joint space are the object of the optimization process. Contrarily, if contact free motion is observed, the optimization process focuses on both hand motion in Cartesian space and joint positions in joint space, and different priorities can be set. The approach exploited in this research is elaborated in Section 4. Conversion from human to humanoid motion is analyzed for a complex task that consists of both types of motion. A transition strategy for motion with and without contact is introduced. Since the task is motion imitation with contact between hands and equipment, the technique proposed by Ude is unable to generate these types of motions for the robot. The advantage of the proposed conversion algorithm over existing algorithms is precise imitation of the position and orientation of human hand motions, which is necessary to perform the task. The results of the conversion algorithm were tested on the ROMEO robot in the same environment as that of the human.

The following paragraphs present kinematic parameters and their correlations for each model instance in the conversation process (human and graphical/digital representation - avatar, scaled model of the humanoid, and ROMEO). An overview of the entire human-to-humanoid motion conversion process is provided in Section 2. Each step of the conversion process is explained in detail.

Fig. 1. Models in the human-to-humanoid motion conversation process and related parameters

The conversion process for human-to-humanoid motion imitation is directly related to the transformation process from kinematic human parameters to those of the humanoid. To that end, three kinematic models were used: kinematic model of the human graphical/digital representation (avatar), scaled kinematic 
model of the ROMEO robot, and kinematic model of the real-size ROMEO robot (see Fig. 1). These models were represented by their parameters in Cartesian space and joint space, as indicated in Fig. 1. The conversion process started with the kinematic model of the human body, which was automatically generated by the motion capture system. This digital/graphical representation of the human body was denoted as the avatar model. The avatar model contained twenty spherical joints (60 degrees of freedom (DoFs) in total), to define its kinematic model. The avatar segment sizes which corresponded to those of the human were estimated by the motion capture system. To enable human motion imitation by a humanoid robot, ROMEO was selected because it is a humanoid with 37 DoFs, but its segment sizes differ from those of a human. In order to make a connection between the avatar and the humanoid kinematic model, an intermediate model, called a scaled model of the humanoid, was defined. The scaled model of the humanoid had the same kinematic structure as ROMEO (37 DoFs) and the same segment sizes as those in the avatar model.

The conversion from the avatar to the model of the humanoid in Cartesian and joint spaces was described by equations, to facilitate understanding of the scaling process. Considering the avatar model, Eq. (1) relates generalized joint coordinates $\left(q_{h}\right)$ and hand position and orientation in Cartesian space:

$$
X_{h}=J_{h} q_{h}
$$

For dual-arm manipulation, $X_{h}$ represents the position and orientation of both human hands, and $J_{h}$ is the Jacobian matrix of the avatar model. The dimension of vector $q_{h}$ contains 60 elements that correspond to the avatar's 60 DoFs. However, to enable conversion from the recorded human motion to the generated ROMEO robot motion, the scaled kinematic model of ROMEO was used to represent the human body model. To that end, the scaled robot model had 37 DoFs and segment dimensions equal to human. The imitation algorithm, based on the scaled model of the humanoid, was defined with the ultimate goal of 
generating human-like motion of the humanoid in joint space. The marker trajectories and the joint positions in Cartesian space obtained for the avatar model were translated to the scaled model of the humanoid. Consequently, this information was input into the imitation algorithm to obtain the human motion in joint space $q_{s r}$. Accordingly, the human hand motions in Cartesian space generated by the scaled model of ROMEO were calculated from the equation:

$$
X_{h}=J_{s r} q_{s r}
$$

where $J_{s r}$ is the Jacobian matrix of the scaled model of ROMEO. For humanoid robot control, the joint motion $q_{s r}$ is useful but the motions of the hand, which are in contact with the environment, are mandatory. Since this information was not directly measured by instrumentation, it was defined via Eq. (2) based on the joint motion resulting from the imitation process and the Jacobian matrix $J_{s r}$. To arrive at human motion of the humanoid robot ROMEO, joint motion $q_{s r}$ can be played on the robot. However, the motion of the robot's hands in Cartesian space, resulting from joint motion $q_{s r}$, was defined by the equation:

$$
X_{\text {robot }}=J_{\text {robot }} q_{s r}
$$

where $J_{\text {robot }}$ is the Jacobian matrix of the ROMEO model and $X_{\text {robot }}$ is the position and orientation of ROMEO's hands in Cartesian space. The scaling factors between models of different segment sizes were integrated through the Jacobian matrices $J_{\text {robot }}$ and $J_{s r}$. The motion of the robot's hands in Cartesian space ( $X_{\text {robot }}$ ) was different from the motion of human hands $X_{h}$ since the segment sizes of the human and the robot were not the same. Therefore, the motion of the robot's hands generated in this manner can only be used for a motion without contact between hands and equipment. If the motion involves such a contact, the motion of the robot's hands has to be the same as that of the human in Cartesian space: 


$$
X_{\text {robot }}=X_{h}
$$

Thus, in contact tasks where the motion of the robot's hands has to be the same as of human hands in Cartesian space, and where, additionally, human motion needs to be imitated in joint space, the generalized robot coordinates $\left(q_{\text {robot }}\right)$ can be defined by the inverse kinematic algorithm (5). The inverse kinematic algorithm is the object of optimization, as explained in Section 4.

$$
q_{\text {robot }}=J_{\text {robot }}^{+} X_{h}+\left(I-J_{\text {robot }}^{+} J_{\text {robot }}\right) q_{s r}
$$

Here, $J_{\text {robot }}^{+}$represents the pseudo-inverse of the matrix $J_{\text {robot }}$ and $I$ is the identity matrix.

The paper is organized as follows. Section 2 presents the conversion steps from human to humanoid motion and the basic characteristics of the robot and the capture motion system. Section 3 proposes our imitation algorithm that converts data obtained from the capture motion system into joint trajectories of the scaled robot model. The imitation process starts with the definition of the humanoid robot kinematic model. The motion generation algorithm for the real robot ROMEO, which takes into account the output from the imitation algorithm, is given in Section 4. The conversion process was tested for the dual arm “open/close drawer" task and the results are presented in Section 5.

\section{Conversion Steps from Human to Humanoid Motion}

Regardless of the motion capture system, the conversion from human to humanoid motion can be represented in four steps: the first step is recording of human motion; in the second, a digital/graphical representation of the human body (avatar) is defined according to the data acquired by the motion capture system; in the third step a human-size kinematic model of the robot (scaled humanoid model) is defined and the imitation algorithm used to obtain motions in the joint and Cartesian spaces of the humanoid; and the 
fourth step concerns the generation of ROMEO's motion (with and without contact), using the output from the imitation algorithm. An overview of the conversion from human to humanoid motion is given in Fig. 2. 


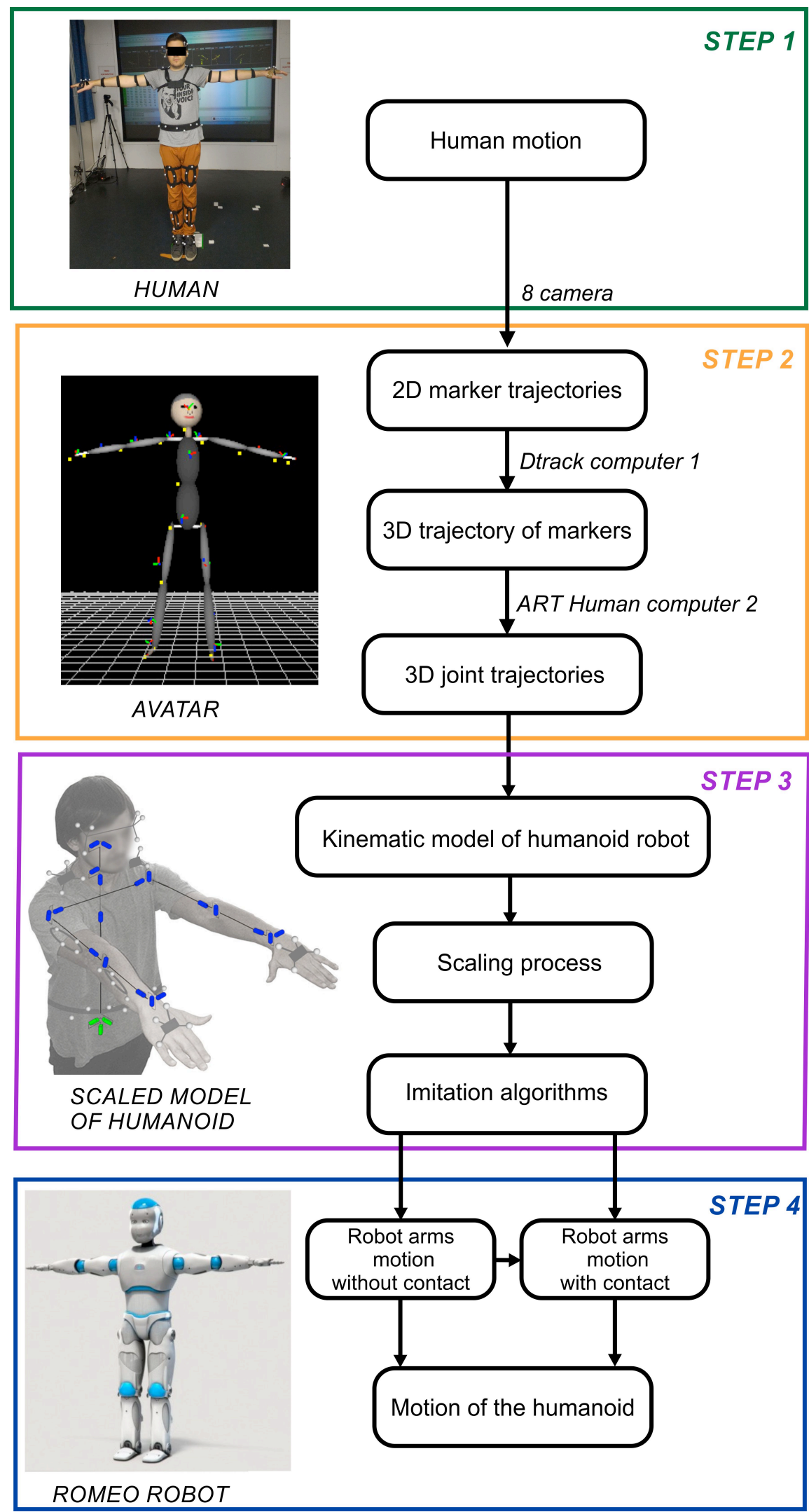

Fig. 2. The process of conversion from human to humanoid motion 
The motion capture system is able to recognize and find relations between the actor's body segments in consecutive frames and provide a graphical representation of the actor (avatar) in a virtual environment [19] (see Fig. 2, step 1). The kinematic model of the avatar graphically represented the human body and included 60 DoFs (20 joints with 3 DoFs per joint). Since the kinematic model of the humanoid, which was used in the imitation process, had 37 DoFs in total (fewer DoFs than the avatar), the model of the avatar could not be used directly and a reduced model that enabled human to humanoid motion replication had to be adopted.

An adult human body has 206 bones linked by different types of joints, which can be flexible. The imitation process needs to be based on the effective kinematic model of the humanoid robot, consistent with its joint mobility and size. Many humanoid robots of a size close to that of a human have several DoFs, e.g. at least 7 DoFs per arm and 6 DoFs per leg (see Fig. 3). A humanoid robot with these characteristics, such as the humanoid robot ROMEO, can be used for accurate human motion imitation. The closer the kinematic model of the humanoid is to the model of a human as regards the size, joint limits and kinematic model, the better the imitation.

The transformation of the kinematic model of the avatar to the model of ROMEO is described on two levels. At the first level, the simplification of the kinematic model was taken into account, so the kinematic model of the avatar (which had 60 DoFs) was reduced to the model of the robot ROMEO (37 DoFs). This kinematic model, which had the same segment size as that of the avatar model and the number of DoFs equal to those of ROMEO, was defined as a scaled model of ROMEO. Therefore, the scaled model of ROMEO was used in the imitation algorithm to define human motion in joint space. The output of the imitation algorithm was coherent motions in joint space and Cartesian space for the scaled model of ROMEO. At the second level, the difference in body segments between the human and ROMEO was taken into account in order to imitate human motion with the real model of ROMEO. Imitation was 
implemented with the algorithm for human-like motion generation. At this level, the motion defined in joint and Cartesian spaces provided by the imitation algorithm was non-coherent (using the real size of ROMEO) and the algorithm favored the motion defined in joint or Cartesian space depending on whether the task did or did not involve contact with the environment.

\subsection{Robot ROMEO}

The humanoid robot ROMEO has been developed by Aldebaran Robotics (see Fig. 3). ROMEO is 1.4 meters tall and has 37 DoFs, including 7 DoFs per arm, 6 DoFs per leg, 2 DoFs for each eye, 1 DoF for each foot, 2 DoFs for the neck, 2 DoFs for the head, and 1 DoF for the spine [20].

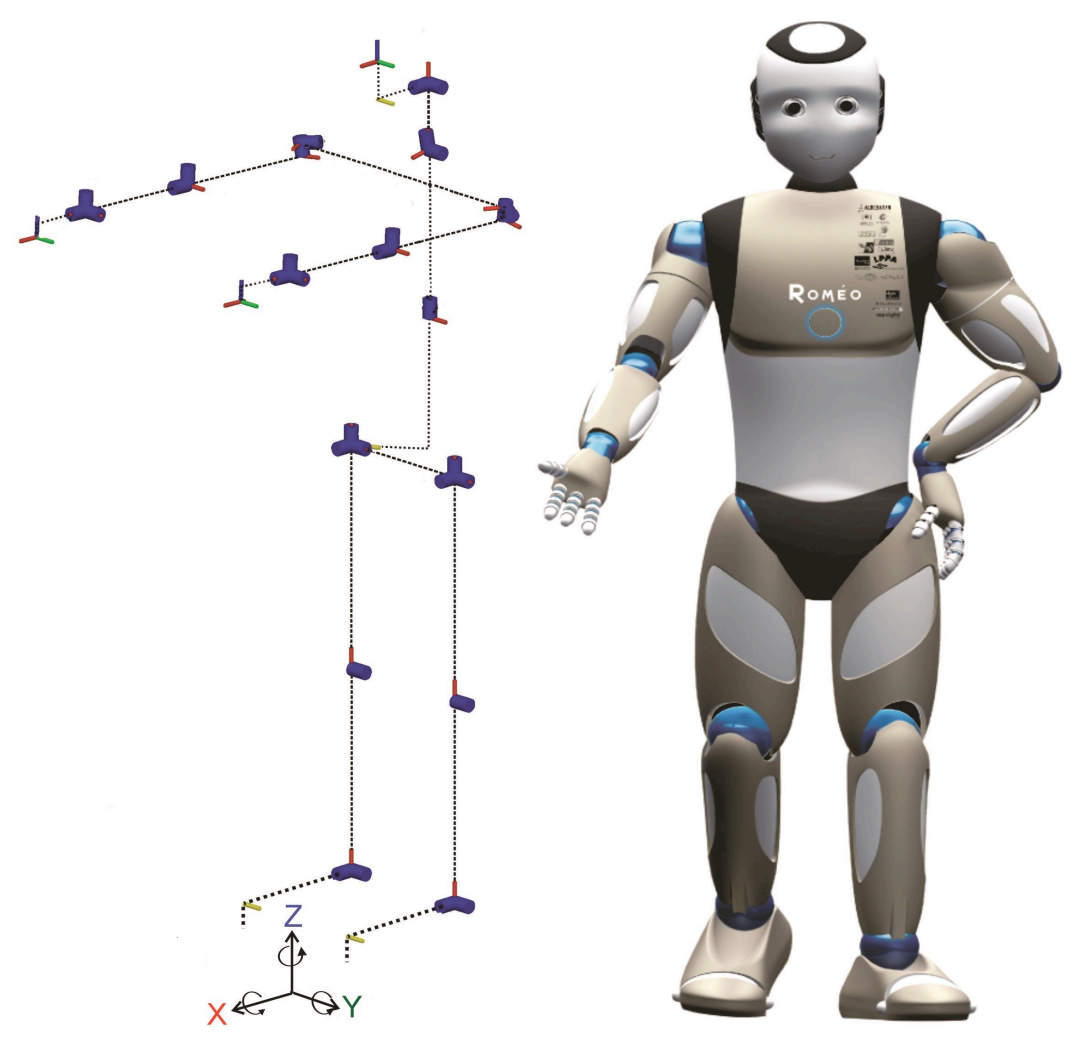

Fig. 3. Robot ROMEO and its kinematic model 
Dual arm manipulation of the robot was analyzed in this research. To that end, an extended kinematic model of ROMEO's upper body was used. The model contained 19 DoFs and an additional 3 DoFs in the trunk, which emulated leg motion (3 prismatic (see Fig. 4)).

\subsection{ART Motion Capture System}

The ART motion capture system consists of a hybrid suit of 17 sets of markers relative to the feet, shins, thighs, shoulders, upper arms, forearms, hands, head, hip, back, and torso (see Fig. 2). A set of 8 infrared (IR) cameras is used to record markers motions. The motion capture hardware is supported by DTrack software and ART Human software is used for tracking and reproducing the motion of the actor in a virtual environment. DTrack software acquires 2D marker positions using information from each IR camera and provides the transformation matrices of each marker (position and orientation of the marker) relative to different local frames attached to the body parts with respect to the global reference frame. ART Human software uses the information provided by DTrack and provides a direct link between motion of the human subject and motion of the 3D graphical model of the human (avatar) in real-time. ART Human estimates the locations of the human joint frames and the human segment size using marker information. The digital human representation, the avatar model, is created in this manner [19]. The flowchart showing how the motion capture system works is depicted in Fig. 2, step 2. The sampling frequency for data acquisition is set to $100 \mathrm{~Hz}$.

\section{The Imitation Process}

The information provided by the motion capture system can be used to define the characteristics of the human body and its motion in the task space. In the previous decades, the focus of some research papers 
has been on estimating a kinematic model of the human body using data obtained by the motion capture systems [21, 22]. In this research, we used data obtained by the ART motion capture system to scale the kinematic model of the robot ROMEO to the characteristics of the actor.

The motion capture system always automatically generates the avatar model as a 3D graphical representation of the human. Each avatar joint is represented as spherical. Therefore, the avatar model has 60 DoFs to represent human motion. The kinematic model of ROMEO was used to imitate human motion in joint space. Therefore, the kinematic representation of the human body was reduced from the avatar model to the kinematic model of ROMEO. Since the information obtained from the motion capture system (such as the position and orientation of real markers and the position of joints) is defined according to the model of the human-size avatar performing the recorded action, the kinematic model of ROMEO was scaled to the size of the human. In that manner it was possible to calculate human motion in joint space.

An initialization process was introduced with the aim of defining the initial configuration of the actor and to scale the size of ROMEO to the size of the actor and attach virtual markers to the scaled model of ROMEO. The initial configuration of ROMEO (see Fig. 4(a)) is proposed as the initial configuration of the actors (arms horizontally extended forward, with palms facing the floor (see Fig. 4(b))), because it is well defined and easily achievable. 
Figure 4. The scaling process: (a) extended kinematic model of the robot ROMEO in its basic configuration; (b) extended kinematic model of ROMEO scaled to the dimensions of a human in the initial configuration, with markers and marker frames

Since the kinematic model of ROMEO was taken as the kinematic model of a human, the size of the robot's segments had to be scaled to the size of the actor's limbs. The dimensions of the human segments were calculated by taking the mean Euclidian distance between two adjacent joints and using several samples from the recorded data, when the actor retained the initial configuration. The dimensions of the paired segments located on the left and right sides of the body, assumed to be identical, were calculated by taking the mean value of the estimated segment dimensions on the right and left sides for each actor. The scaling factor $S F$ for each segment was calculated as the ratio of the actor segment length $L_{\text {human }}$ to ROMEO segment length $L_{\text {robot }}, S F=\frac{L_{\text {human }}}{L_{\text {robot }}}$

The position of the real marker relative to the corresponding proximal frame of the actor's joint and the dimensions of the segments were calculated and assumed constant in the scaled model of the humanoid. In reality, this quantity varies during motion of the actor due to unmodeled joints of the human and motion of the skin. The unmodeled joints in the shoulders are responsible for vertical displacement of the shoulders 
and the real markers attached to the shoulders (see Fig. 5). Although the markers are firmly attached to the body segments, the distance between a real marker and its proximal joint is not constant due to the motion of the skin. This phenomenon is the most obvious in the case of hand and shoulder markers. An error occurs and a perfect imitation of human motion by the scaled kinematic model of the humanoid is not possible. Virtual marker fames were defined according to real marker frames in the initial configuration, during the initialization process. Details about the calculation of virtual markers frames are provided in the Appendix. The imitation algorithm was used to calculate the joint configuration of the scaled model of ROMEO, in order to minimize the matching error between the virtual and real markers and the matching error between the joint positions of the human to the joint positions of the scaled model of ROMEO.

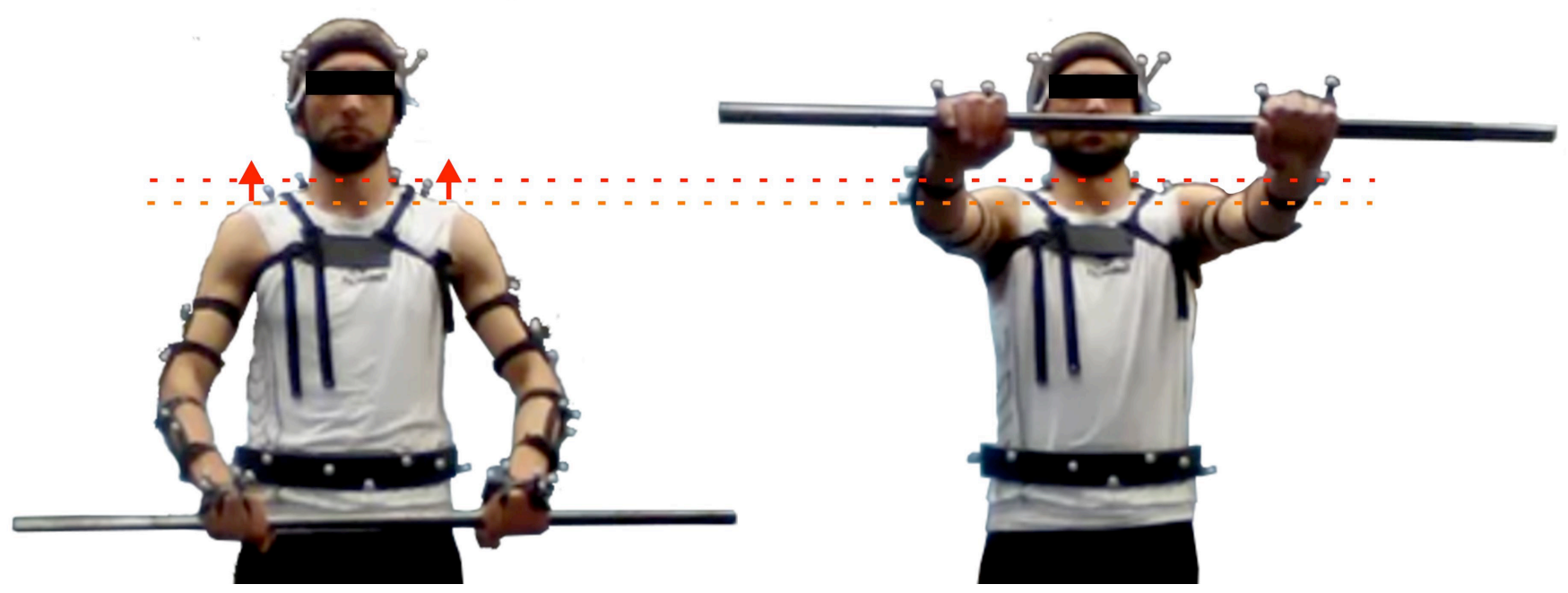

Figure 5. Displacement of the shoulders and real markers attached to the shoulders during motion.

\subsection{Imitation algorithm for the scaled model of the robot}

In this section, an analytical imitation algorithm based on the Jacobian matrix capable of real time extraction of Cartesian motions and joint motions is introduced. The imitation algorithm was formulated as an optimization algorithm (6), which calculated the generalized coordinates of the joints $q_{\text {imitation }}\left(\mathrm{t}_{i}\right)$ for the scaled model of ROMEO, for each time sample $t_{i}$, with $i \in\left[\begin{array}{ll}1 & N\end{array}\right]$, where $N$ is the number of time samples of the recorded motion. 


$$
q_{\text {imitation }}\left(t_{i}\right)=\min _{q}(\zeta)
$$

The criterion function $\zeta$, which should be minimized, was comprised of the error between the position of the frames attached to the actor joint $P_{a j}$ and those of the scaled model of the humanoid $P_{r j}$; the error between the position of the real marker frames $P_{r m}$ and virtual marker frames $P_{v m}$; and the error in orientation between the real marker frames $R_{r m}$ and virtual marker frames $R_{v m}$ in the way proposed in Eqs. (7) and (8). Since the precision of orientation measurement was lower than that of position measurement, and the orientations of the proximal segments were implicitly taken into account via their effect on distal joint positions and markers, the orientations of the proximal frames were disregarded in the minimization criterion. Only the orientations of the distal segments were included.

The optimization criterion is:

$$
\zeta=\left\|\varepsilon\left(t_{i}, q\right)\right\|^{2}
$$

where

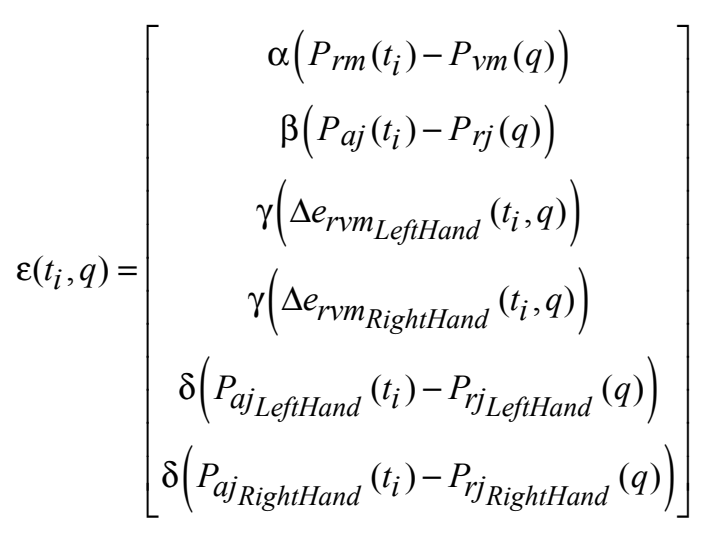

where $\alpha, \beta, \gamma$ and $\delta$ are the weighting factors for the errors in marker positions, joint positions, hand marker orientations, and hand joint positions, respectively; $P_{r m}\left(t_{i}\right)$ and $P_{a j}\left(t_{i}\right)$ are the vectors of the recorded real marker positions and proximal actor joints at time sample $t_{i}$, respectively; $P_{v m}(q)$ and $P_{r j}(q)$ are the vectors of the positions of the virtual markers and proximal robot joints in the current joint configuration $q$ : 


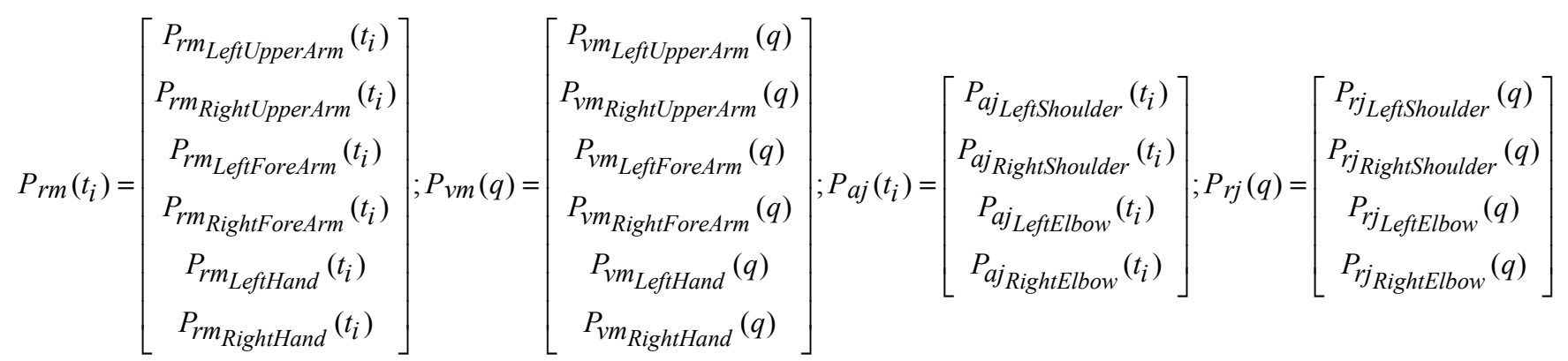

$P_{a j_{\text {LeftHand }}}\left(t_{i}\right)$ and $P_{a j_{\text {RightHand }}}\left(t_{i}\right)$ are the positions of the left and right actor hand joints at time sample $t_{i}$,

respectively; $P_{r j_{\text {LeftHand }}}(q)$ and $P_{r j_{\text {RightHand }}}(q)$ are the positions of the left and right robot hand joints in the current joint configuration $q$; and $\Delta e_{r v m_{\text {LeftHand }}}$ and $\Delta e_{r v m_{\text {RightHand }}}$ are the orientation errors between the real and virtual markers attached to the left and right hands (distal markers) at time sample $t_{i}$ and in joint configuration $q$, respectively. The orientation errors were represented in terms of quaternions. The rotation matrices, $R_{r m_{\text {LeftHand }}}\left(t_{i}\right)$ and $R_{v m_{\text {LeftHand }}}(q)$ (which express the real and virtual marker orientations of the left hand, respectively), can be rewritten in terms of quaternions as

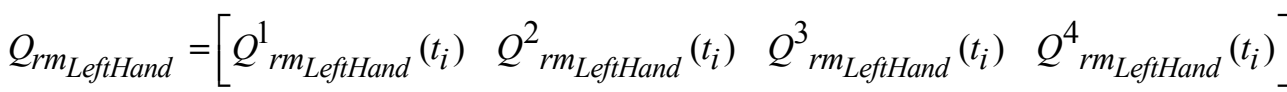
and $Q_{v m_{l}}=\left[\begin{array}{llll}Q_{v m_{\text {LeftHand }}}^{1}\left(t_{i}\right) & Q_{v m_{\text {LeftHand }}}^{2}\left(t_{i}\right) & Q_{v m_{\text {LeftHand }}}^{3}\left(t_{i}\right) & Q_{v m_{\text {LeftHand }}}^{4}\left(t_{i}\right)\end{array}\right] \quad, \quad$ respectively $\quad$ where

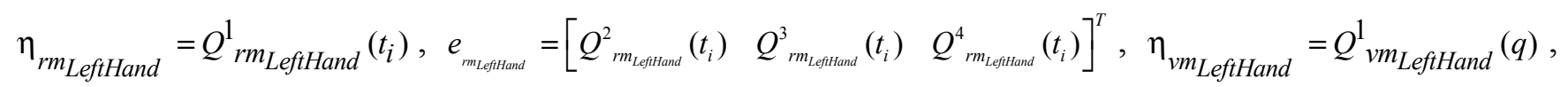

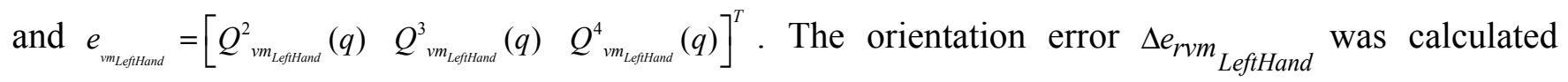
using Eq. [22]:

$$
\begin{aligned}
& \Delta e_{r v m_{\text {LeftHand }}}\left(t_{i}, q\right)=\eta_{v m_{\text {LeftHand }}}(q) \cdot e_{r m_{\text {LeftHand }}}\left(t_{i}\right)- \\
& \eta_{r m_{\text {LeftHand }}}\left(t_{i}\right) \cdot e_{v m_{\text {LeftHand }}}(q)-S\left(e_{r m_{\text {LeftHand }}}\left(t_{i}\right)\right) \cdot e_{v m_{\text {LeftHand }}}(q)
\end{aligned}
$$

where $S(\cdot)$ is the skew-symmetric operator of the vector.

The orientation error of the right hand $\Delta e_{r v m_{\text {RightHand }}}$ was calculated in the same way. 
The minimization criterion is quadratic. The configuration at time sample $t_{i}$ can be expressed as the configuration at time $t_{i-1}$ plus the change in configuration $\Delta q$. Thus, the criterion can be expressed as a function of $\Delta q$ :

$$
\varepsilon\left(t_{i}, q\right)=\varepsilon\left(t_{i}, q_{\text {imitation }}\left(t_{i-1}\right)+\Delta q\right)=\varepsilon_{\alpha}\left(t_{i}, q_{\text {imitation }}\left(t_{i-1}\right)\right)+\varepsilon_{\beta}\left(t_{i}, q_{\text {imitation }}\left(t_{i-1}\right)\right) \Delta q
$$

where vector $\varepsilon_{\alpha}\left(t_{i}, q_{\text {imitation }}\left(t_{i-1}\right)\right)$ can be evaluated based on Eq. (8), and matrix $\varepsilon_{\beta}\left(t_{i}, q_{i m i t a t i o n}\left(t_{i-1}\right)\right)$ based on its derivative. The analytical expression for $\Delta q$ is deduced for the optimality condition

$$
\begin{array}{r}
\frac{\partial \zeta}{\partial \Delta q}=\frac{\partial\left(\left\|\varepsilon\left(t_{i}, q_{\text {imitation }}\left(t_{i-1}\right)+\Delta q\right)\right\|^{2}\right)}{\partial \Delta q}=0, \text { so that: } \\
\qquad q=-\varepsilon_{\beta}\left(t_{i}, q_{\text {imitation }}\left(t_{i-1}\right)\right)^{+} \cdot \varepsilon_{\alpha}\left(t_{i}, q_{\text {imitation }}\left(t_{i-1}\right)\right)
\end{array}
$$

where $\varepsilon_{\beta}\left(t_{i}, q_{\text {imitation }}\left(t_{i-1}\right)\right)^{+}$represents the pseudo inverse of the matrix $\varepsilon_{\beta}\left(t_{i}, q_{\text {imitation }}\left(t_{i-1}\right)\right)$. More details about vectors $\varepsilon_{\alpha}\left(t_{i}, q_{\text {imitation }}\left(t_{i-1}\right)\right)$ and matrix $\varepsilon_{\beta}\left(t_{i}, q_{\text {imitation }}\left(t_{i-1}\right)\right)$ are provided in the Appendix.

Factors $\alpha, \beta, \gamma$ and $\delta$ in the imitation algorithm assigned different desired levels of precision for joint and marker tracking. The values of the weighting factors were set heuristically. Since contact tasks require high precision of the hand positions and orientations in Cartesian space, weighting factors $\delta$ (hand position weighting factor) and $\gamma$ (hand marker orientation) had significantly higher values than other factors. However, the weighting factor $\gamma$ should be set to slightly less than $\delta$, since orientation measurement precision is lower than that of position measurement. The positions of proximal (shoulder and elbow) joints in Cartesian space is less important than the Cartesian position of the hand joints, but they still have a significant effect on the similarity between recorded human motion and generated humanoid motion. The associated weighting factor was $\beta$. The similarity between the recorded human motion and the generated humanoid motion was depicted by marker position as well, but with the aim of reducing the effect of skin motion (which directly influences the motion of the markers), weighting factor $\alpha$ had to be set to the lowest value. Accordingly, the weighting factors were selected as follows: $\alpha=1, \beta=2, \gamma=10$ 
and $\delta=20$.

The actor motion in Cartesian space $X_{h}$ was re-calculated using $q_{\text {imitation }}\left(\mathrm{t}_{i}\right)$ and the direct geometric scaled model of the ROMEO robot. Furthermore, the orientation of the hands' joints, which was unknown from the data obtained by the motion capture system, was also calculated in this manner. A comparative analysis of the recorded hand positions and the position calculated by the imitation algorithm is given in Section 5.

\section{From Imitation Results to Motion of Robot ROMEO}

The motion obtained with the previously described imitation algorithm could not be used directly by the humanoid robot ROMEO because joint limitations had not been taken into account. If joint motion $q_{\text {imitation }}(t)$ is used directly by ROMEO, the motion in Cartesian space will not be preserved and thus contact with the environment will not be achieved. On the other hand, if Cartesian motion is used, human skill will not be preserved. Therefore, we propose different strategies depending of the existence of contact with the environment or not. A contact with the environment is assumed to exist via the hands of the robot. The case of contact with another body part may be considered in a similar way. A transition strategy will also be proposed between the contact and no contact phases.

During a motion with contact, the motion of the actor's hands was constrained by the characteristics of the equipment. In [24], we elaborated the types of contact constraints and the corresponding mathematical representations. Here we studied the case in which the robot should do the task in the same environment as that of humans, with the same type of contact. The moment when the robot's hands established contact with the equipment was calculated using the hand positions of the scaled model of the robot and the known position of the equipment. To that end, the hand coordinates of the scaled robot model and the equipment coordinates were represented in the same referent frame. For the phase of contact, the robot's hands should be able to follow the same motion in Cartesian space as the actor's hands. A necessary condition for 
performing the task by the robot is that the trajectories of the actor's hands (for the phase of contact) are within the workspace of the robot. The workspace of ROMEO was defined according to the robot segment size and the joint limits as proposed in $[25,26]$. If this condition is satisfied, when the robot is initially in the same place as the actor, the actor's motion will be easily achieved by ROMEO. Otherwise, a new initial position and orientation of the robot need to be calculated for the task to become feasible. In the latter case, the transformation matrix that describes the displacement of the robot is taken into account, in order to modify the desired motion of the robot's hands accordingly.

\subsection{Inverse kinematic algorithm as a tool for human-like motion generation}

The human motion imitation process requires the robot to be able to perform a task like a human. Since the structure of ROMEO is designed with redundant features, the same motion as that of human hands can be obtained for different arm configurations. By using the inverse kinematic algorithm, the recorded human joint motion can be imitated by ROMEO. The minimized difference between the current joint trajectories $q_{\text {robot }}(t)$ and joint trajectories $q_{\text {imitation }}(t)$ obtained with the imitation algorithm for the scaled robot model $\left|q_{\text {robot }}(t)-q_{\text {imitation }}(t)\right|^{2}$ was included in the inverse kinematic algorithm as an additional criterion:

$$
\begin{aligned}
& d q_{\text {robot }}\left(t_{i}\right)=J_{\text {robot }}+\lambda d X\left(t_{i}\right)-\left(I-J_{\text {robot }}{ }^{+} J_{\text {robot }}\right)\left(q_{\text {robot }}\left(t_{i-1}\right)-q_{\text {imitation }}\left(t_{i}\right)\right) \\
& q_{\text {robot }}\left(t_{i}\right)=q_{\text {robot }}\left(t_{i-1}\right)+d q_{\text {robot }}\left(t_{i}\right)
\end{aligned}
$$

where $J_{\text {robot }}+\lambda$ is the damped least-square inverse of the Jacobian matrix $J_{\text {robot }}$ and damping factor $\lambda=0.003$ (see [27]), which was introduced with the aim of solving the discontinuity problem of the pseudoinverse solution for a singular configuration; $J_{\text {robot }}{ }^{+}$is the pseudo-inverse of the Jacobian matrix $J_{\text {robot }}$ of ROMEO, calculated for the robot size; $I$ is the identity matrix; and $d X\left(t_{i}\right)$ is the position and orientation variation between the desired trajectories of the robot's hands $X_{\text {robot }}^{d}\left(t_{i}\right)$ and the current position and orientation of the robot's hands $X_{\text {robot }}^{c}\left(q_{\text {robot }}\left(t_{i-1}\right)\right)$, calculated by the direct geometric model 
using the real size of the robot:

$$
d X\left(t_{i}\right)=X_{\text {robot }}^{d}\left(t_{i}\right)-X_{\text {robot }}^{c}\left(q_{\text {robot }}\left(t_{i-1}\right)\right)
$$

The desired trajectories of the robot's hands $X_{\text {robot }}^{d}$ have to be the same as the motion of human hands in Cartesian space $X_{h}$. The joint limits were included in the inverse kinematic algorithm by using the internal clamping loop that checked and removed the joints that reached their upper or lower joint limits $q_{\text {robot }}^{\min }$ and $q_{\text {robot }}^{\max }$, respectively. If a joint reached the limit, the joint value was clamped to the limit value. The elements of the Jacobian matrix $J$ and identity matrix $I$ related to the clamped joint were set to zero. In this manner, any further motion of the clamped joint is prevented. The inverse kinematic algorithm continues to initiate other joints in order to reach the desired values for the hands. The inverse kinematic algorithm with a clamping loop is explained in detail in Baerlocher et al. [28].

The primary task of the inverse kinematic algorithm was to follow the desired hand trajectories $X_{\text {robot }}^{d}\left(t_{i}\right)$. If motion involved contact between hands and equipment, the robot needed to use the same equipment and manipulate it in the same way as a human. The desired motions of the robot's hands were defined in the imitation algorithm, calculating the hand position and orientation using $q_{\text {imitation }}(t)$ and the direct geometric model of the scaled ROMEO. However, it is important to note that $q_{\text {imitation }}(t)$ was defined for a scaled model of the robot and that Jacobian matrix $J$ used here was calculated for the real size of ROMEO. Parameters $q_{\text {imitation }}(t)$ and $X_{\text {robot }}^{d}\left(t_{i}\right)$ were not consistent, thus the secondary task was not achieved and $q_{\text {robot }}(t)$ differed from $q_{\text {imitation }}(t)$.

\subsection{Motion of robot hands without contact}

A robot is not able to simultaneously follow recorded hand and joint motions since the robot size and joint limits and those of a human are not the same. When there is no contact with the environment, it may be 
preferable to follow human joint motions rather than human Cartesian motions, in order to express human skills.

However, to keep the same control approach with and without contact with the environment, and to take into account the joint limits, we used a slightly modified Eq. (12):

$$
d X\left(t_{i}\right)=X_{\text {robot }}^{c}\left(q_{\text {imitation Modif }}\left(t_{i}\right)\right)-X_{\text {robot }}^{c}\left(q_{\text {robot }}\left(t_{i-1}\right)\right)
$$

where $q_{\text {imitationModif }}\left(t_{i}\right)$ is the modified value of $q_{\text {imitation }}(t)$, since the value of $q_{\text {imitation }}(t)$ can be outside the robot's joint limits. The modification was implemented using the algorithm proposed by Safonova et al. [29]. In this manner, $X_{\text {robot }}^{c}\left(q_{\text {imitation Modif }}\left(t_{i}\right)\right)$ was inside the workspace of the robot and the desired motion in the joint and Cartesian spaces was coherent and consistent with the dimensions of the humanoid robot. The joint limits were also taken into account.

\subsection{Transition strategy connecting motions with and without contact}

For a motion with or without contact of the hands with the environment, we proposed the algorithm given by Eqs. (12) and (14). The difference between the two cases is in the manner used to define the desired Cartesian motion. As a consequence, we propose a transition strategy based on rescaling the size of the robot to the size of the actor. The transition strategy starts during the motion without contact, if the position of the robot's hands relative to the object to be contacted reaches a prescribed vicinity. Here we assumed the prescribed vicinity was a sphere of $0.1 \mathrm{~m}$ radius. During transition, the sizes of the robot segments on the model were linearly modified to reach the sizes of the actor. The hand trajectories were calculated for the incrementally rescaled model of the robot by using the value of $q_{\text {imitationModif }}\left(t_{i}\right)$, which corresponds to this part of the motion. The transition strategy was over when the size of the rescaled model of the robot was the same as that of the actor and the value of $q_{\text {imitationModif }}\left(t_{i}\right)$ was the same as that corresponding to the sample when the contact between the actor's hands and the equipment was achieved. 
A connection between the hands and the equipment was therefore made.

The desired trajectories of the robot's hands can be processed further. In order to smooth trajectories of the robot's hands, we applied the Savitzky-Golay filter which is based on fitting consecutive sub-sets of adjacent data points with a low-degree polynomial by the linear least squares method [30].

\subsection{Handling collision}

This research also addressed dual-arm motions performed by actors. Any actor naturally avoids collision with equipment and self-collision. Since we used data generated by the imitation algorithm, which characterize human skills, to generate motion of the robot it is expected that the robot, similarly to the actor, will avoid self-collision. Also, the trajectories of the robot's hands during the contact phase of the motion were the same as those of a human, thus collision with the equipment was eliminated. Collisions did not occur in the tested motion. In other cases, collision avoidance could be included in the generation of humanoid motion using the techniques developed in [31-33]. The vision-based path planning method for dual-arm end-effector features proposed by Qu et al. [34] is also a useful tool for precise pose alignment of two objects, which is the position and orientation of the hands and equipment in our case.

\section{Results}

In this section we will analyze the results of the imitation algorithm and the conversion process for the "open/close drawer" task. "Open/close drawer" is a complex motion which consists of motions with and without contact. Initially, the motion was without contact until the actor touched the drawer. The task continued with a motion with contact, during which the actor performed the drawer opening and closing functions. In order to show the general characteristics of the imitation algorithm, we tested it on a set of 7 different dual-arm tasks, each performed by 19 actors, and present the corresponding results. The results obtained with the imitation algorithm are compared with those of the numerical algorithm proposed by Ude $[13,14]$. The imitation algorithm proposed by Ude is a numerical optimization approach based on 
following the 3D position of markers physically attached to the human body, with markers placed on the scaled robot model. That imitation algorithm has been developed for imitating free human motion (motion without contact) and, consequently, precise imitation of the hand joints was not required.

\subsection{Simulation results of the imitation algorithm}

The imitation algorithm proposed in this paper was designed to give the highest priority to hand position and orientation tracking, to ensure that the task was properly done. According to the results for the "open/close drawer" task presented here (see Fig. 6, color blue), our imitation algorithm produced the same motion of the actor's hands as the scaled model of the ROMEO robot. The highest normed errors in following the actor's hand trajectories were about $4 \mathrm{~mm}$. 

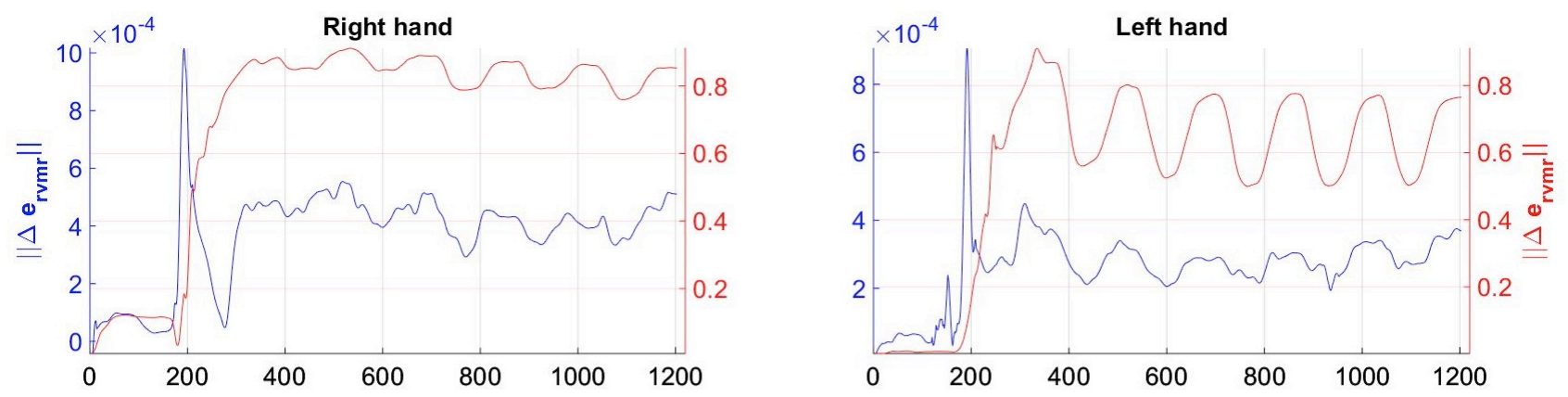

a)
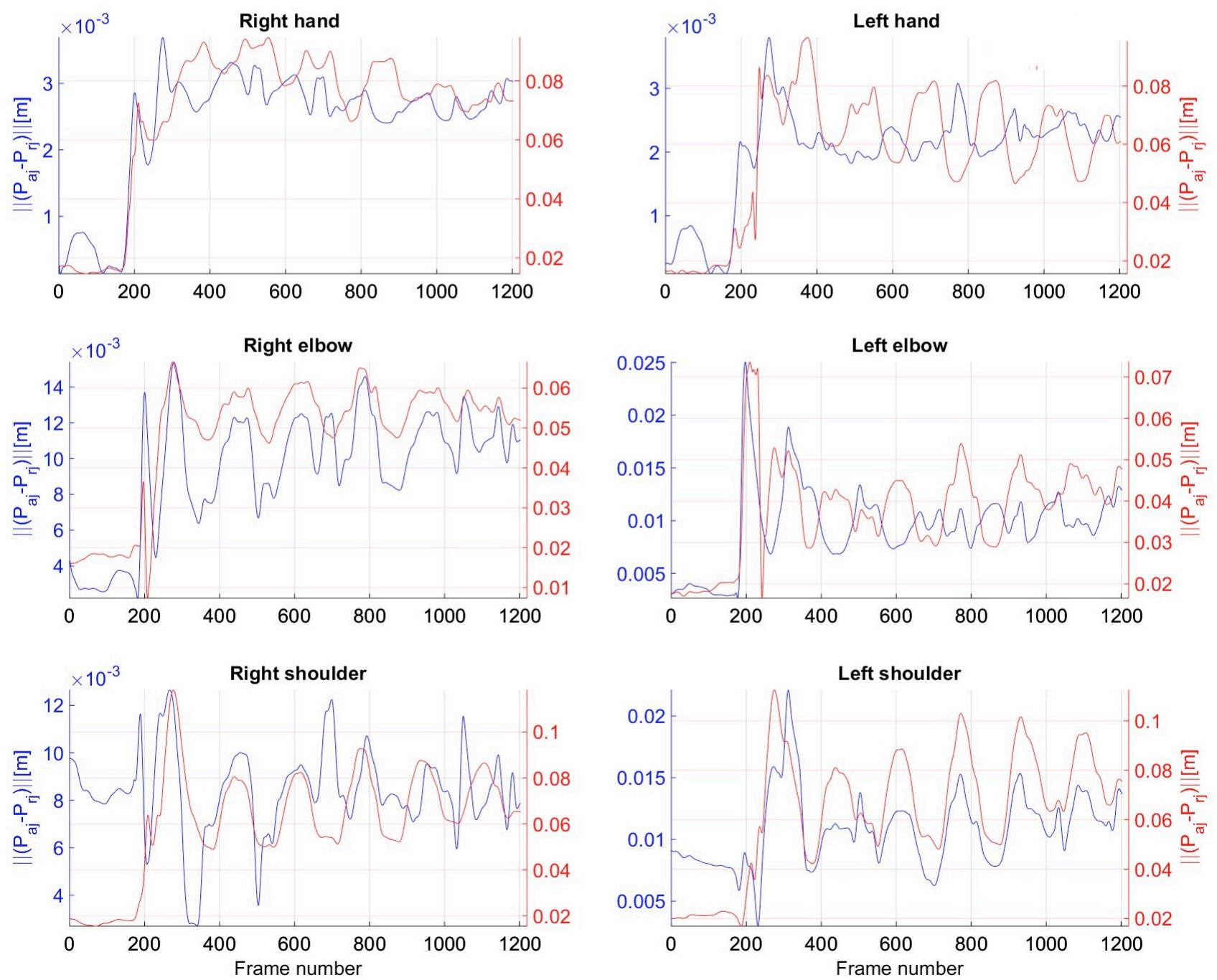

b)

Figure 6. Orientation and position errors between the recorded motion and the motion generated by our (analytical) imitation algorithm (blue scale) and the numerical imitation algorithm proposed by Ude [13] (red scale): (a) normed orientation errors $\Delta e_{r v m_{\text {RightHand }}}$ and $\Delta e_{r v m_{\text {LeftHand }}}$ of the right and left hand markers in quaternion; and (b) normed position errors $P_{a j}\left(t_{i}\right)-P_{r j}\left(t_{i}\right)$ of the arm joints in meters. 
Since the robot model had enough DoFs, the imitation algorithm performed well when following hand orientations. Bigger errors in following the shoulder and elbow joint motions (larger amplitude around 25 $\mathrm{mm}$ ) were the results of simplifying the kinematic model of the human by using the model of the robot ROMEO. To highlight the performance of the proposed imitation algorithm, the results are compared with the numerical algorithm proposed by Ude et al. $[13,14]$ and shown in Fig. 6, red scale. Since Ude's numerical algorithm is based on following 3D marker positions, the errors in following the joint position and orientation are bigger compared to our imitation algorithm (see Fig. 6). In [14], big errors in following hand joint position and orientation would not allow ROMEO to achieve contact between hands and equipment and the task would not be accomplished. On the other hand, errors in following the position of the real marker with the virtual marker obtained with the algorithm proposed by Ude are smaller compared with the results of our imitation algorithm, since hand position tracking is of the highest priority in our algorithm (see Fig. 7).
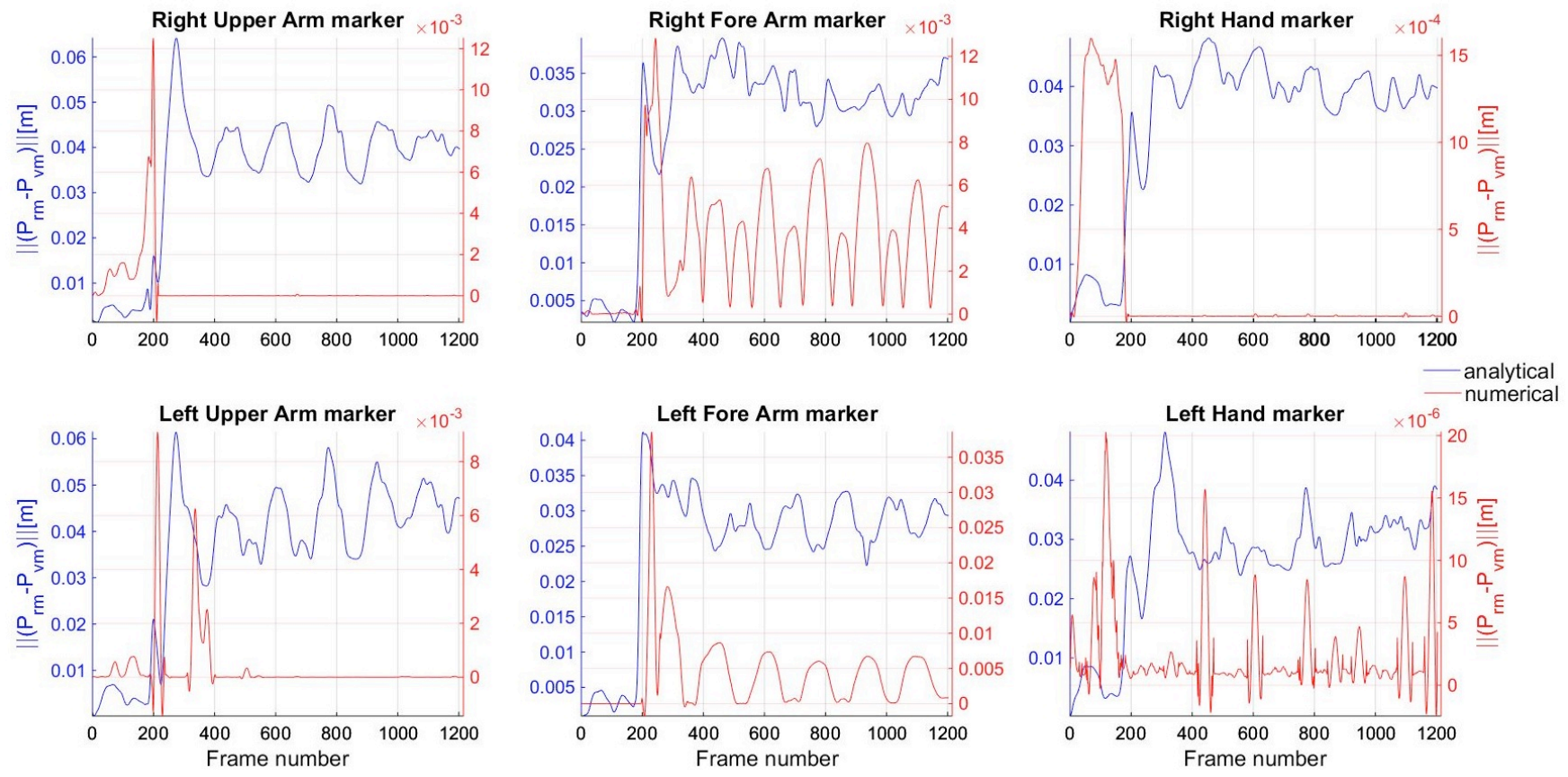

Fig. 7. Normed position errors in following real marker $P_{r m}$ with virtual marker $P_{v m}$ obtained with our (analytical) imitation algorithm (blue scale) and the numerical imitation algorithm proposed by Ude [13] 
(red scale), in meters.

Although the algorithm proposed by Ude ensures a small error when the 3D position of the markers is followed, precise following of the position and orientation of the hand joints is not guaranteed. The reasons are the calculation of the transformation matrix between real markers and the closest proximal joint, which is not so precise, and the fact that this transformation matrix may change due to skin motion. It is apparent that our algorithm allows better tracking of the hand motion compared with the algorithm proposed by Ude and also ensures correct tracking to the pose of the shoulder and elbow that characterize the shape of the arm (i.e. its configuration).

Summing up, the hand trajectories generated through our imitation algorithm can be used as a desired robot motion in the conversion process, in the inverse kinematic algorithm for the task with and without contact between hands and equipment.

The imitation algorithm was tested on a set of different dual-arm motions ("rotation of valves", "rotation of canoe paddles", "inflating a mattress with a pump", "open/close drawer", "rotation of a steering wheel", "cutting with knife" and "grating food"), each performed by 19 actors. The quality of imitation of one motion was represented as $\mathrm{E}=\mu\left(d_{P_{a_{\text {hand }}}\left(t_{i \ldots N}\right), P_{r_{\text {hand }}}\left(t_{i \ldots N}\right)}\right)$, the mean value of the Euclidian distance between the recorded positions of the hand joints $P_{a_{\text {hand }}}$ and those obtained with our imitation algorithm $P_{r_{h a n d}}$ for all samples of the motion. In order to present the general performance of the imitation algorithm for each actor, we calculated $\mu(E)$ and the mean value of $E$ for all the motions performed by each actor. The general characteristics of our imitation algorithm for each actor expressed by $\mu(E)$ and the imitation algorithm proposed by Ude are shown in Fig. 8. 


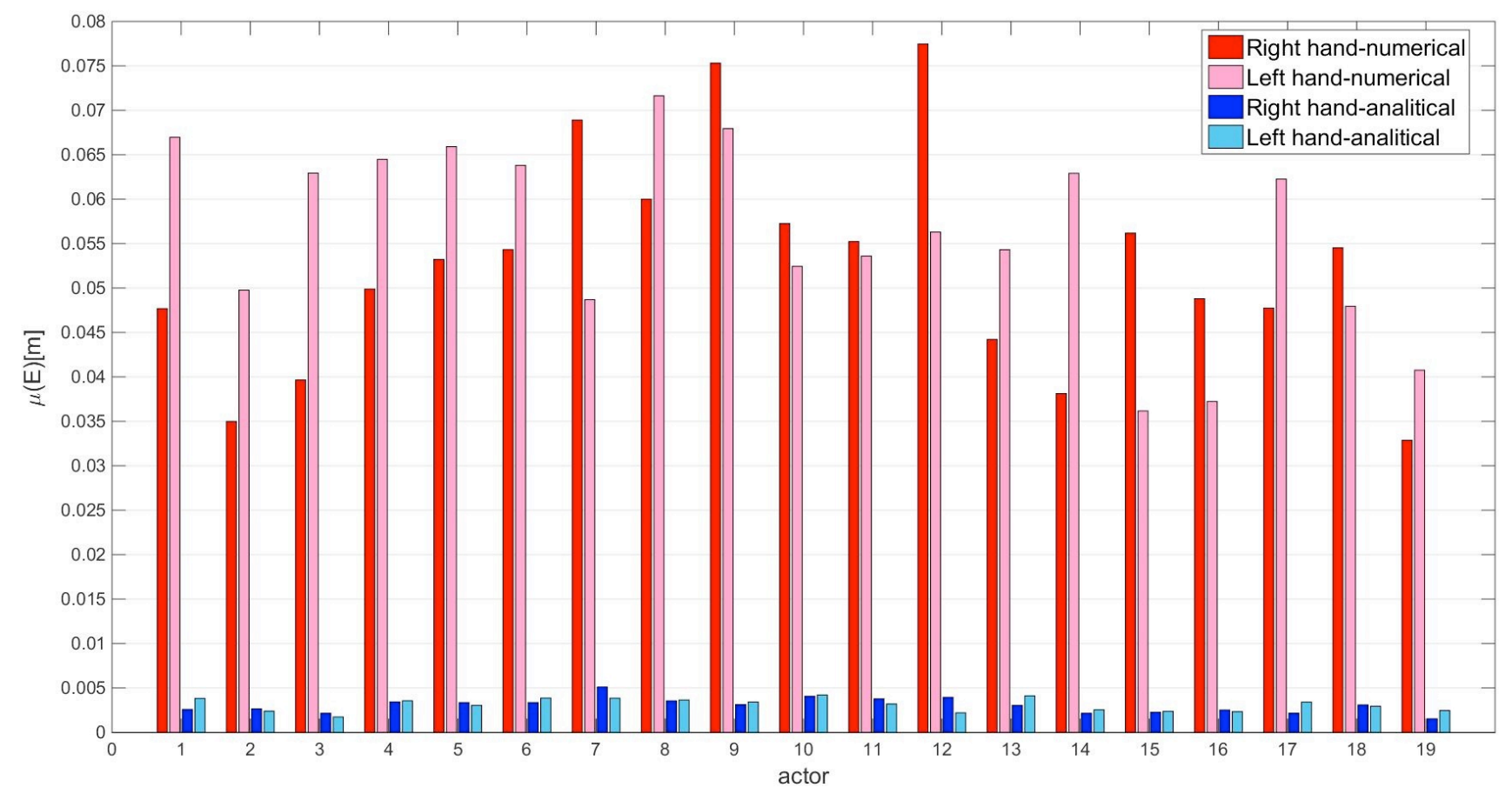

Figure 8. Quality of imitations of right and left hand motions of 19 actors, expressed by $\mu(E)$, obtained by our (analytical) algorithm (blue) and the numerical algorithm proposed by Ude (red).

The results show that our imitation algorithm has average errors in following the desired hand positions of about $3 \mathrm{~mm}$ for most actors. For some actors, these errors are somewhat bigger, about $5 \mathrm{~mm}$. The reason is a large disparity between the simplified human model represented by ROMEO and the kinematic model of the real actor (confidence in the distance between joints).

\subsection{ROMEO robot motion simulation and experimental results}

The conversion process is based on the results obtained from the imitation algorithm, which are applied in the algorithm for humanoid motion generation. The trajectories of the hand motions obtained for the scaled robot model by using the imitation algorithm are shown in Fig. 9(b). Since the size of the robot was not equivalent to the size of the actor, the desired hand trajectories during the motion without contact were outside the robot's workspace and the robot was unable to perform the motion. Therefore, the hand motions generated with the imitation algorithm had to be additionally modified according to the robot's 
characteristics, as proposed in our algorithm for humanoid motion. The generated trajectories of the robot's hands during the motion with and without contact, as well as the transition strategy, are shown in Fig. 9(c).

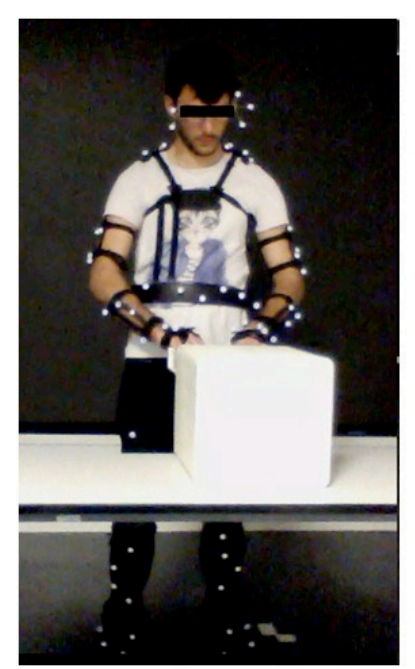

a)

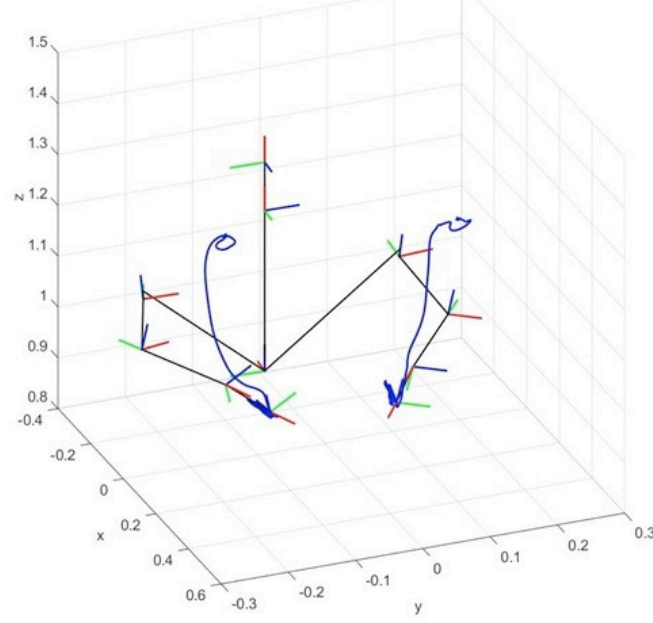

b)

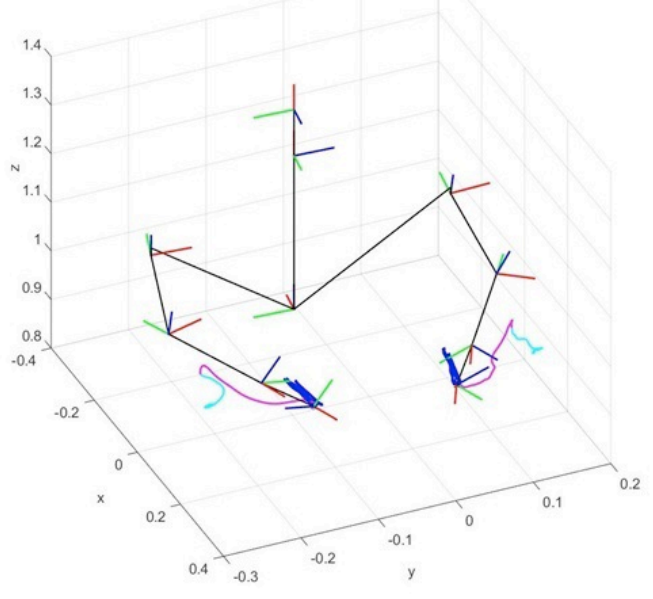

c)

Figure 9. "Open/close drawer" task performed by the actor and the robot: (a) Actor during the task: simulation model of the robot and calculated trajectories of the robot's hands, (b) in the imitation algorithm, and c) in the algorithm for humanoid motion generation.

The trajectories depicted in cyan are the motions without contact with equipment, obtained as proposed in Section 4.2; the trajectories in magenta represent the motions obtained with the transition strategy when the contact between the hands and equipment was calculated according to the algorithm proposed in Section 4.3; and dark blue represents the trajectories of the actor's hands during motion with contact. The desired trajectories of the robot's hands are based on the results of the actor's motion from the imitation algorithm and/or the motion of the robot's arms imitating human motion. Therefore, the robot's hand motion was free of both collision with equipment and self-collision, like the recorded human motion. The experimental results of the actor's "open/close drawer" motion and the same motion performed by ROMEO are shown in Fig. 10. The robot and the actor performed the task in the same environment. The height of the robot was less that the height of the actor. Consequently, the robot performed the task at the 
level of its chest, whereas the actor did so at waist level. ROMEO had conventional hands with flexible fingers, which were not actuated and the robot was required to just open and close hands. This was the reason why the drawer was ajar at the beginning of the experiment. With a robot that has complex hands with actuated joints, the contact between the hands and the drawer would have been possible with the drawer closed, exactly in the same manner as the actor performed the task.
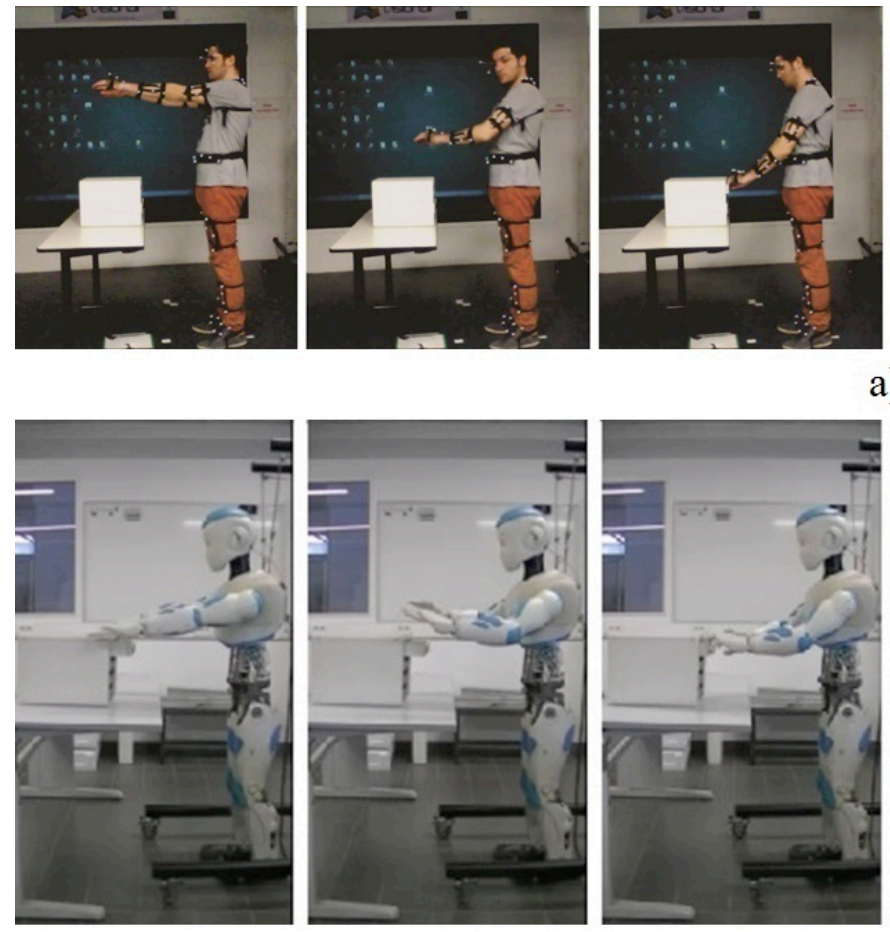

a)

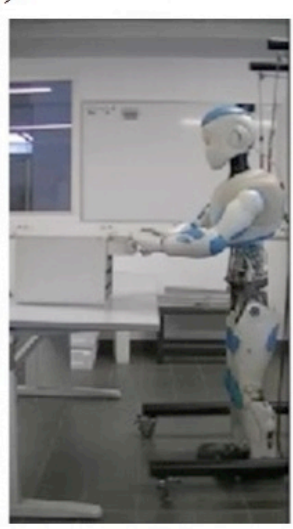

b)
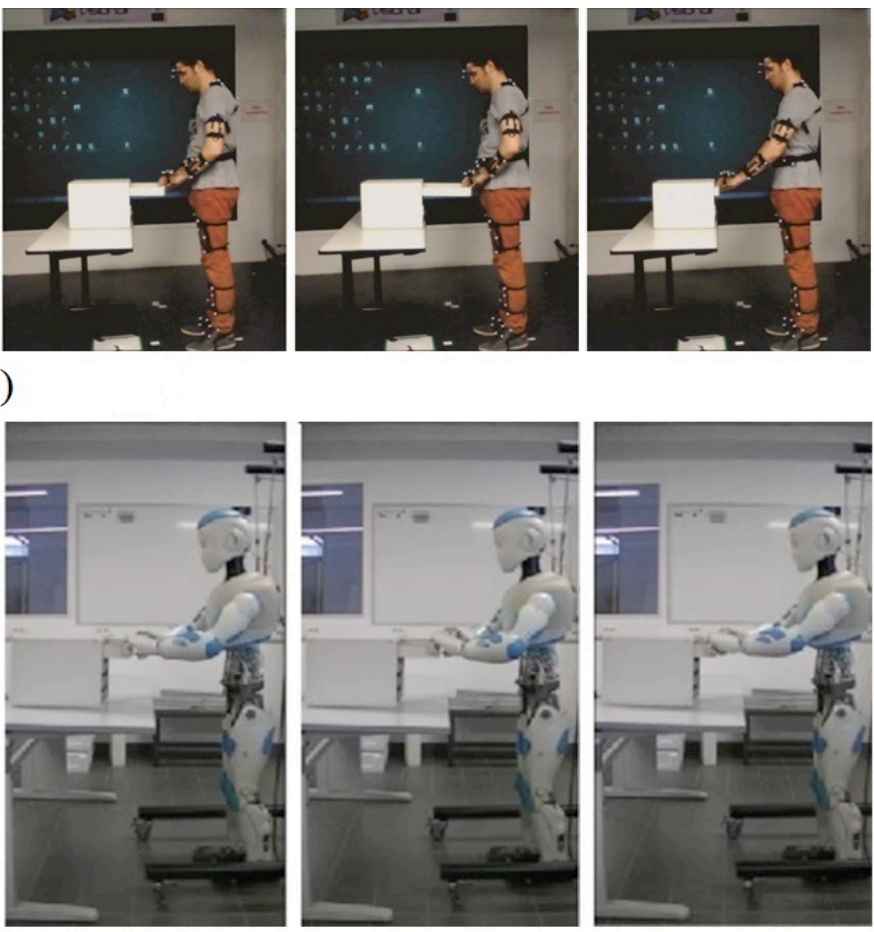

Fig 10. Snapshot of the "open/close drawer" motion performed by: (a) the actor; (b) and the robot

ROMEO. The robot was able to perform the same task under the same conditions as the actor.

\section{Conclusion}

A conversion process for the imitation of dual-arm human motion, utilizing the upper body, has been presented. It consists of an imitation algorithm and an algorithm for humanoid motion generation. The imitation algorithm, defined for a scaled model of the robot ROMEO, is based on virtual markers which follow real marker motions and incorporates additionally recorded joint motions. The imitation algorithm 
is based on the analytical expression of Jacobian matrices and is able to define the expected motion of the scaled robot in real time. Compared to existing algorithms, our imitation algorithm provides better accuracy of motion imitation in Cartesian space. Precise imitation of hand motion in Cartesian space is essential for a task where the hands come into contact with the environment. The algorithm for humanoid motion generation is based on an inverse kinematic algorithm whose objective is to follow the desired robot hand motions and, at same time, ensure that the motion of the humanoid resembles human motion behavior. Since our task involved motion which included phases with and without contact between hands and equipment, we additionally defined an algorithm for the transition between the phases. Therefore, by way of an important contribution of this work, the proposed conversion algorithm is suitable for human motion imitation by a humanoid for a task with and without contact, as well as complex tasks that involve both types of motion, which other imitation algorithms are not. The results of our conversion process were experimentally tested on the real robot ROMEO. Ultimately, one can say that the proposed conversion methodology can be used as a universal and robust algorithm for human-to-humanoid motion conversion, regardless of the type of dual-arm motion or the characteristics of the actor.

\section{Acknowledgements}

The research leading to these results has received funding from the Ministry of Education, Science and Technological Development of the Republic of Serbia, under contract TR-35003, and the Equipex Robotex project funded by CNRS under grant agreement No. ANR-10- EQPX-44-01.

\section{References}

1. M. Do, P. Azad, T. Asfour and R. Dillmann, "Imitation of human motion on a humanoid robot using non-linear optimization," In: Proceeding of the Humanoids 2008-8th IEEE-RAS International Conference on Humanoid Robots (2008) pp. 545-552. 
2. D. Vlasic et al, "Practical motion capture in everyday surroundings," ACM transactions on graphics (TOG) 26(3), 35 (2007).

3. N. Miller, O. C. Jenkins, M. Kallmann and M. J. Mataric, "Motion capture from inertial sensing for untethered humanoid teleoperation," In: Proceeding of the Humanoid Robots, 2004 4th IEEE/RAS International Conference on IEEE (2004) 2 pp. 547-565.

4. S. Aloui, C. Villien, and S. Lesecq, “A new approach for motion capture using magnetic field: models, algorithms and first results," International Journal of Adaptive Control and Signal Processing 29(4), $407-426(2015)$.

5. E. Ceseracciu, Z. Sawacha, and C. Cobelli, "Comparison of markerless and marker-based motion capture technologies through simultaneous data collection during gait: proof of concept," PloS one 9(3), e87640 (2014).

6. H. Zhou, and H. Hu, "Human motion tracking for rehabilitation-A survey," Biomedical Signal Processing and Control 3(1), 1-18 (2008).

7. M. J. Gómez, C. Castejón, J. C. Garcia-Prada, G. Carbone and M. Ceccarelli, “Analysis and comparison of motion capture systems for human walking," Experimental Techniques 40(2), 875-883 (2016).

8. A. Billard et al, "Discovering optimal imitation strategies," Robotics and autonomous systems 47(2), 69-77 (2004).

9. C. Ott, D. Lee, and Y. Nakamura, "Motion capture based human motion recognition and imitation by direct marker control,” In: Proceedings of the IEEE-RAS International Conference on Humanoid Robots (2008) pp. 399-405.

10. W. Suleiman et al, "On human motion imitation by humanoid robot," In: Proceedings of the IEEE International Conference on Robotics and Automation (ICRA 2008) (2008) pp. 2697-2704.

11. Q. Huang, Z. Yu, W. Zhang, W. Xu and X. Chen, "Design and similarity evaluation on humanoid motion based on human motion capture." Robotica 28(05), 737-745 (2010). 
12. R.S. Jamisola, P.S. Kormushev, R.G. Roberts, et al., "Task-space modular dynamics for dual-arms expressed through a relative Jacobian,” Journal of Intelligent \& Robotic Systems 83(2), 205-218 (2016). https://doi.org/10.1007/s10846-016-0361-0

13. A. Ude, C.G. Atkeson and M. Riley, "Programming full-body movements for humanoid robots by observation,” Robot. Auton. Syst. 47(2), 93-108 (2004).

14. A. Ude, C. Man, M. Riley and C.G. Atkeson, "Automatic Generation of Kinematic Models for the Conversion of Human Motion Capture Data into Humanoid Robot Motion," In: Proceeding of the First IEEE-RAS International Conference on Humanoid Robots (2000) pp. 2223 -2228.

15. K. Ayusawa, Y. Ikegami and Y. Nakamura, "Simultaneous global inverse kinematics and geometric parameter identification of human skeletal model from motion capture data," Mechanism and Machine Theory 74, 274-284 (2014).

16. K. Ayusawa, M. Morisawa and E. Yoshida, "Motion retargeting for humanoid robots based on identification to preserve and reproduce human motion features," In: Proceeding of the Intelligent Robots and Systems (IROS) (2015) pp. 2774-2779.

17. M. Tomić, C. Vassallo, C. Chevallereau, et al., "Arm Motions of a Humanoid Inspired by Human Motion," In New Trends in Medical and Service Robots. Springer International Publishing pp. 227- 238 (2016).

18. W. Khalil and J. Kleinfinger, "A new geometric notation for open and closed-loop robots," Robotics and Automation. Proceedings. 1986 IEEE International Conference on 3, 1174-1179 (1986).

19. ART GmbH, System user manual ARTtrack, TRACKPACK and DTrack, 2015, version 2.11.: http://http://www.schneider-digital.com/support/download/Tools-Ressourcen/ARTTracking/Dokumentat ion/ARTtrack DTrack TrackPACK User Manual 2.11.pdf/(last visited 2nd November 2016.)

20. FUI national Romeo project: http://projetromeo.com (last visited 2nd November 2016.)

21. H. Köhler, M. Pruzinec, T. Feldmann, and A.Worner, “Automatic Human Model Parametrization from 
3D Marker Data for Motion Recognition," In: International Conference in Central Europe on Computer Graphics, Visualization and Computer Vision (2008).

22. A. G. Kirk, J. F. O'Brien, and D. A. Forsyth, "Skeletal Parameter Estimation from Optical Motion Capture Data," In: Proceedings of the IEEE Computer Society Conference on Computer Vision and Pattern Recognition (2005) 2 pp. 782-788.

23. B. Siciliano, L. Sciavicco, L. Villani, and G. Oriolo, "Robotics: modelling, planning and control," Springer Science and Business Media (2010).

24. K. Jovanović, V. Potkonjak, O. Holland, "Dynamic modeling of an anthropomimetic robot in contact tasks," Advanced Robotics 28(11), pp. 793-806 (2014).

25. M. Bagheri, "Kinematic analysis and design considerations for optimal base frame arrangement of humanoid shoulders," In: 2015 IEEE International Conference on Robotics and Automation (ICRA) (2015) pp. 2710-2715.

26. P. Wenger, "Performance Analysis of Robots," Modeling, Performance Analysis and Control of Robot Manipulators 141-183 (2010).

27. C. W. Wampler, "Manipulator inverse kinematic solutions based on vector formulations and damped least-squares methods," IEEE Transactions on Systems, Man, and Cybernetics 16(1), 93-101 (1986).

28. P. Baerlocher and R. Boulic, "An inverse kinematics architecture enforcing an arbitrary number of strict priority levels," The visual computer 20(6), 402-417 (2004).

29. A. Safonova, N. Pollard, and J. K. Hodgins, "Optimizing human motion for the control of a humanoid robot," Proc. Applied Mathematics and Applications of Mathematics 78 (2003).

30. S. J. Orfanidis, "Introduction to Signal Processing," Prentice-Hall (1996).

31. M. Mühlig, M. Gienger, and J. J. Steil, "Interactive imitation learning of object movement skills," Autonomous Robots 32(2), 97-114 (2012).

32. B. Dariush et al, "Online transfer of human motion to humanoids," International Journal of Humanoid 
Robotics 6(02), 265-289 (2009).

33. M. Ruchanurucks, "Humanoid robot upper body motion generation using B-spline-based functions," Robotica 33(04), 705-720 (2015).

34. J. Qu, F. Zhang, Y. Fu, and S. Guo, "Multi-cameras visual servoing for dual-arm coordinated manipulation,” Robotica 35(11), 2218-2237 (2017). doi:10.1017/S0263574716000849

35. W. Khalil, and D. Creusot, "SYMORO+: a system for the symbolic modelling of robots," Robotica 15, 153-161 (1997).

\section{Appendix}

The imitation algorithm proposed in this paper addresses an optimization problem which has an analytical solution for the value $q_{\text {imitation }}\left(t_{i}\right)$ at each time sample $t_{i}$, minimizing the criterion function given by Eq. (7). Since the initial configuration of the robot, $q_{\text {init }}$, is calculated in the initialization process and the initial positions of the robot's joints and virtual markers are known, the current value $q_{\text {imitation }}\left(t_{i}\right)$ can be calculated incrementally by using $q_{\text {imitation }}\left(t_{i-1}\right)$ calculated in the previous iteration $q_{\text {imitation }}\left(t_{i}\right)=q_{\text {imitation }}\left(t_{i-1}\right)+\Delta q$. Therefore, the criterion function, $\varepsilon\left(q\left(t_{i}\right)\right)$, can be expressed as a function of $\Delta q$ :

$$
\begin{aligned}
& \varepsilon\left(t_{i}, q\left(t_{i}\right)\right)=\varepsilon\left(t_{i}, q_{\text {imitation }}\left(t_{i-1}\right)+\Delta q\right)=
\end{aligned}
$$

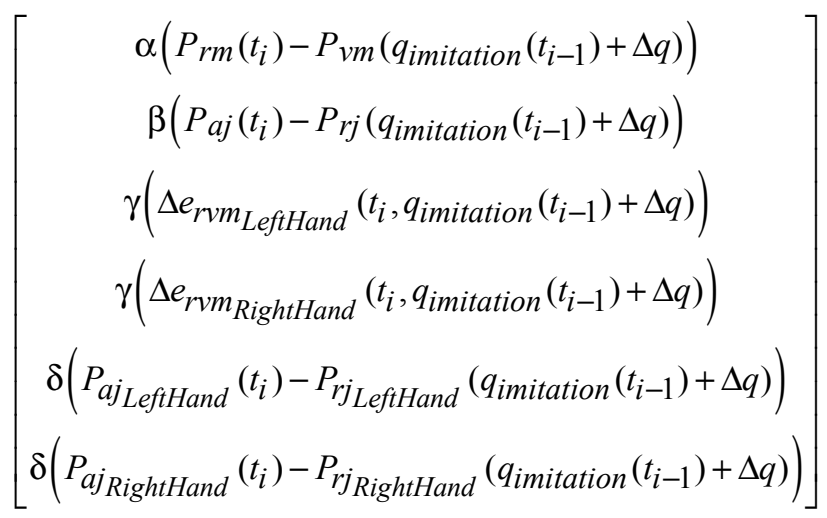

The current position of the $k^{\text {th }}$ robot joint is represented by the relation

$$
P_{r j_{k}}\left(q\left(t_{i}\right)\right)=P_{r j_{k}}\left(q_{\text {imitation }}\left(t_{i-1}\right)\right)+J_{r j_{k}}\left(q_{\text {imitation }}\left(t_{i-1}\right)\right) \Delta q
$$

where $k \in\{$ LeftShoulder RightShoulder LeftElbow RightElbow $\} . J_{r j_{k}}(q)$ is the Jacobian matrix of the 
$k^{\text {th }}$ proximal robot joint, calculated analytically with SYMORO+ software [35]. The current position of the $l^{\text {th }}$ virtual marker is calculated from the equation

$$
P_{v m_{l}}\left(q\left(t_{i}\right)\right)=P_{v m_{l}}\left(q_{\text {imitation }}\left(t_{i-1}\right)\right)+J_{v m_{l}}\left(q_{\text {imitation }}\left(t_{i-1}\right)\right) \Delta q
$$

where $l \in\{$ LeftUpperArm RightUpperArm LeftForeArm RightForeArm LeftHand RightHand $\}$.

$J_{v m_{l}}(q)$ is the Jacobian matrix of the $l^{\text {th }}$ virtual marker calculated from equation

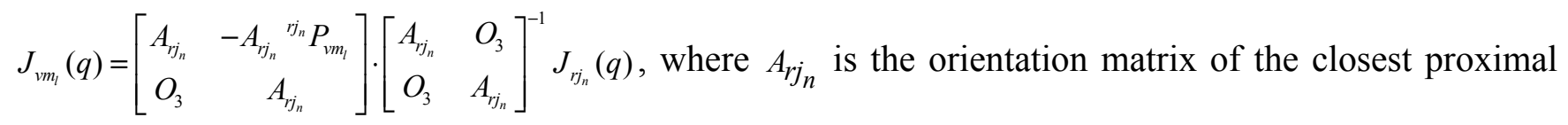
frame attached to joint $T_{r j_{n}}$ via transformation matrix ${ }^{r j_{n}} T_{v m_{l}}$, and ${ }^{r j_{n}} P_{v m_{l}}$ is a skew-symmetric matrix defined by a component of the vector ${ }^{r j_{n}} P_{v m_{l}}$. The current orientation of the virtual markers on the left hand is calculated in the same way as the position

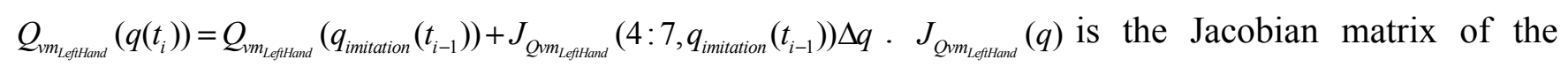
left-hand virtual marker represented in terms of quaternion and calculated using

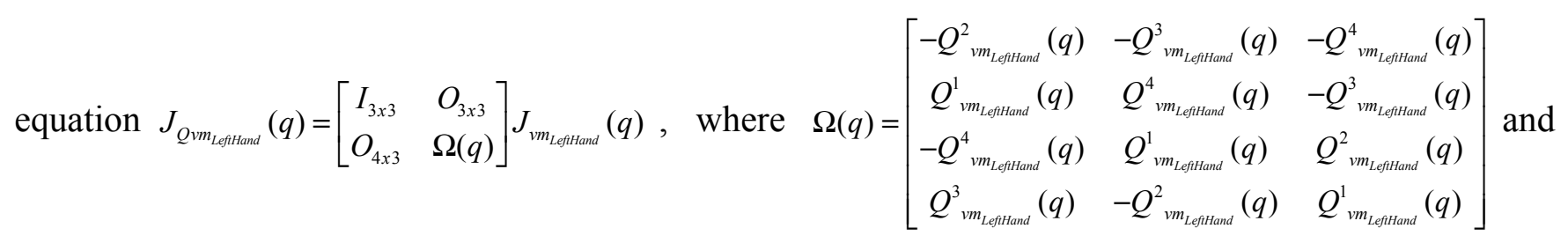

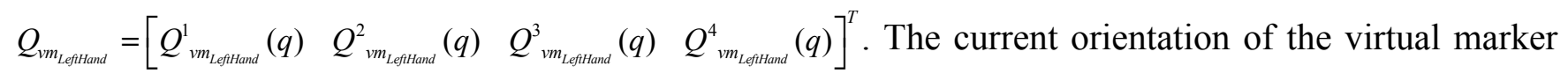
on the right hand is calculated in the same way.

According to the previous equations, vector $\varepsilon_{\alpha}\left(t_{i}, q_{\text {imitation }}\left(t_{i-1}\right)\right)$ and matrix $\varepsilon_{\beta}\left(t_{i}, q_{\text {imitation }}\left(t_{i-1}\right)\right)$ from Eq. (10) take the form: 


$$
\varepsilon_{\alpha}\left(t_{i}, q_{\text {imitation }}\left(t_{i-1}\right)\right)=\left[\begin{array}{c}
\alpha\left(P_{r m}\left(t_{i}\right)-P_{v m}\left(q_{\text {imitation }}\left(t_{i-1}\right)\right)\right. \\
\beta\left(P_{a j}\left(t_{i}\right)-P_{r j}\left(q_{\text {imitation }}\left(t_{i-1}\right)\right)\right. \\
\gamma \cdot \varepsilon_{\alpha_{\text {LeftHand }}^{R}}^{R} \\
\gamma \cdot \varepsilon_{\alpha_{\text {RightHand }}^{R}} \\
\delta\left(P_{a j_{\text {LeftHand }}}\left(t_{i}\right)-P_{r j_{\text {LeftHand }}}\left(q_{\text {imitation }}\left(t_{i-1}\right)\right)\right. \\
\delta\left(P_{a j_{\text {RightHand }}}\left(t_{i}\right)-P_{r j_{\text {RightHand }}}\left(q_{\text {imitation }}\left(t_{i-1}\right)\right)\right.
\end{array}\right]
$$

$$
\varepsilon_{\beta}\left(t_{i}, q_{\text {imitation }}\left(t_{i-1}\right)\right)=\left[\begin{array}{c}
-\alpha \cdot J_{v m}\left(q_{\text {imitation }}\left(t_{i-1}\right)\right) \\
-\beta \cdot J_{r j}\left(q_{\text {imitation }}\left(t_{i-1}\right)\right) \\
\gamma \cdot \varepsilon_{\beta_{\text {LeftHand }}^{R}}^{R} \\
\gamma \cdot \varepsilon_{\beta_{\text {RightHand }}^{R}} \\
-\delta \cdot J_{r j_{\text {LeftHand }}}\left(q_{\text {imitation }}\left(t_{i-1}\right)\right) \\
-\delta \cdot J_{r j_{\text {RightHand }}}\left(q_{\text {imitation }}\left(t_{i-1}\right)\right)
\end{array}\right]
$$

where

$$
\begin{aligned}
& \varepsilon_{\alpha_{\text {LeftHand }}}^{R}=\left(\begin{array}{l}
\eta_{v m_{\text {LeftHand }}}\left(q_{\text {imitation }}\left(t_{i-1}\right)\right) \cdot e_{r m_{\text {LeftHand }}}\left(t_{i}\right)- \\
\eta_{r m_{\text {LeftHand }}}\left(t_{i}\right) \cdot e_{v m_{\text {LeftHand }}}\left(q_{\text {imitation }}\left(t_{i-1}\right)\right)- \\
S\left(e_{r m_{\text {LeftHand }}}\left(t_{i}\right)\right) \cdot e_{v m_{\text {LeftHand }}}\left(q_{\text {imitation }}\left(t_{i-1}\right)\right)
\end{array}\right) \\
& \varepsilon_{\alpha_{\text {RightHand }}^{R}}^{R}=\left(\begin{array}{ll}
\eta_{v m_{\text {RightHand }}}\left(q_{\text {imitation }}\left(t_{i-1}\right)\right) \cdot e_{r m_{\text {RightHand }}}\left(t_{i}\right)- \\
\eta_{r m_{\text {RightHand }}}\left(t_{i}\right) \cdot e_{v m_{\text {RightHand }}}\left(q_{\text {imitation }}\left(t_{i-1}\right)\right)- \\
S\left(e_{r m_{\text {RightHand }}}\left(t_{i}\right)\right) \cdot e_{v m_{\text {RightHand }}}\left(q_{\text {imitation }}\left(t_{i-1}\right)\right)
\end{array}\right) \\
& \varepsilon_{\beta_{\text {LeftHand }}}^{R}=\left(\begin{array}{l}
e_{r m_{\text {LeftHand }}}\left(t_{i}\right) \cdot J_{Q v m_{\text {LeftHand }}}\left(4, q_{\text {imitation }}\left(t_{i-1}\right)\right)- \\
\eta_{r m_{\text {LeftHand }}}\left(t_{i}\right) \cdot J_{Q v m_{\text {LeftHand }}}\left(5: 7, q_{\text {imitation }}\left(t_{i-1}\right)\right)- \\
S\left(e_{r m_{\text {LeftHand }}}\left(t_{i}\right)\right) \cdot J_{Q v m_{\text {LeftHand }}}\left(5: 7, q_{\text {imitation }}\left(t_{i-1}\right)\right)
\end{array}\right) \\
& \varepsilon_{\beta_{\text {RightHand }}^{R}}^{R}=\left(\begin{array}{l}
e_{r m_{\text {RightHand }}}\left(t_{i}\right) \cdot J_{Q v m_{\text {RightHand }}}\left(4, q_{\text {imitation }}\left(t_{i-1}\right)\right)- \\
\eta_{r m_{\text {RightHand }}}\left(t_{i}\right) \cdot J_{Q v m_{\text {RightHand }}}\left(5: 7, q_{\text {imitation }}\left(t_{i-1}\right)\right)- \\
S\left(e_{r m_{\text {RightHand }}}\left(t_{i}\right)\right) \cdot J_{Q v m_{\text {RightHand }}}\left(5: 7, q_{\text {imitation }}\left(t_{i-1}\right)\right)
\end{array}\right)
\end{aligned}
$$


$J_{v m}$ is the vector of the Jacobian matrices for all virtual markers and $J_{r j}$ is the vector of the Jacobian matrices for all the proximal robot joints. $\Delta q$ is calculated according to Eqs. (11), (A4), and (A5). The initial guess for the imitation algorithm at the first sample is taken to be the initial configuration, $q_{\text {init }}$, of the scaled model of the robot. In future samples of the imitation algorithm, the solution from the previous sample $q_{\text {imitation }}\left(t_{i-1}\right)$ is used as the initial guess for the current iteration in the optimization algorithm. The values of the cost function cannot be zero due to large differences between the kinematic models of the human and the humanoid.

The position and orientation of the virtual markers are defined from the scaled model of the robot in the initial configuration. The virtual marker frame (with transformation matrix $T_{v m_{l}}$ ) is attached to the body, where it is fixed and connected with the closest proximal frame attached to the joint $T_{r j_{n}}\left(q_{i n i t}\right)$ via transformation matrix ${ }^{r j_{n}} T_{v m_{l}}$. The virtual marker holds the mean values of the real marker transformation matrices $T_{r m_{l}}\left(t_{i=1 . . n_{\text {init }}}\right)$ in $n_{\text {init }}$ time instances of the actor's initial configuration. A transformation matrix ${ }^{r j}{ }_{n} T_{v m_{l}}$ is calculated for each joint and remains unchanged in the imitation algorithm. 